\title{
SNAKES, LADDERS, AND ISOLAS OF LOCALIZED PATTERNS*
}

\author{
MARGARET BECK ${ }^{\dagger}$, JÜRGEN KNOBLOCH ${ }^{\ddagger}$, DAVID J. B. LLOYD ${ }^{\S}$, BJÖRN \\ SANDSTEDE $₫$, AND THOMAS WAGENKNECHT"
}

\begin{abstract}
Stable localized roll structures have been observed in many physical problems and model equations, notably in the $1 \mathrm{D}$ Swift-Hohenberg equation. Reflection-symmetric localized rolls are often found to lie on two "snaking" solution branches, so that the spatial width of the localized rolls increases when moving along each branch. Recent numerical results by Burke and Knobloch indicate that the two branches are connected by infinitely many "ladder" branches of asymmetric localized rolls. In this paper, these phenomena are investigated analytically. It is shown that both snaking of symmetric pulses and the ladder structure of asymmetric states can be predicted completely from the bifurcation structure of fronts that connect the trivial state to rolls. It is also shown that isolas of asymmetric states may exist, and it is argued that the results presented here apply to $2 \mathrm{D}$ stationary states that are localized in one spatial direction.
\end{abstract}

Key words. Snaking, rolls, localized patterns, Swift-Hohenberg equation

AMS subject classifications. 37C29, 35B32, 37K50

1. Introduction. To motivate the results presented in this paper, we begin with a concrete example, namely the Swift-Hohenberg equation, which is given by

$$
U_{t}=-\left(1+\partial_{x}^{2}\right)^{2} U-\mu U+\nu U^{2}-U^{3}, \quad x \in \mathbb{R} .
$$

We shall focus exclusively on the case $\mu>0$, for which the trivial state $U=0$ is stable, and also keep $\nu>0$ fixed. For appropriate values of the parameters, the Swift-Hohenberg equation admits stable stationary spatially-periodic patterns which we refer to as rolls. In fact, rolls form a one-parameter family, parametrized by their wavelength. We are interested in localized roll patches, such as those shown in Figure 1.1, which can be thought of as subjecting rolls to a localized amplitude modulation.

The bifurcation diagrams shown in Figure 1.1, which have been discussed, for instance, in $[29,44,8,3,14,32,33,34,15,16,24,27]$, exhibit several intriguing features, which are commonly referred to as snaking. There are two intertwined wiggly solution branches that correspond to localized patterns whose width, measured by the $L^{2}$-norm, increases as we move along each branch. In particular, there exists a parameter interval so that (1.1) has infinitely many localized patterns for each $\mu$ in this interval. The end points of the interval correspond to saddle-node bifurcations of the localized states, which are aligned along a well-defined vertical asymptote.

*The authors thank John Burke and Edgar Knobloch for sharing results and thoughts on snaking and ladders.

$\dagger^{\dagger}$ Division of Applied Mathematics, Brown University, Providence, RI 02912, USA (margaret_beck@brown.edu). This author was partially supported by the NSF under grant DMS-0602891.

¥Institute of Mathematics, Technical University Ilmenau, 98684 Ilmenau, Germany (juergen.knobloch@tu-ilmenau.de). This author's visit to the UK in Autumn 2007 was supported by a scheme-4 grant of the London Mathematical Society.

$\S$ Department of Mathematics, University of Surrey, Guildford, GU2 7XH, UK (d.j.lloyd@surrey.ac.uk).

"Division of Applied Mathematics, Brown University, Providence, RI 02912, USA (bjorn_sandstede@brown.edu). This author gratefully acknowledges a Royal Society Wolfson Research Merit Award.

"Department of Mathematics, University of Leeds, Leeds, LS2 9JT, UK (thomas@maths.leeds.ac.uk). 

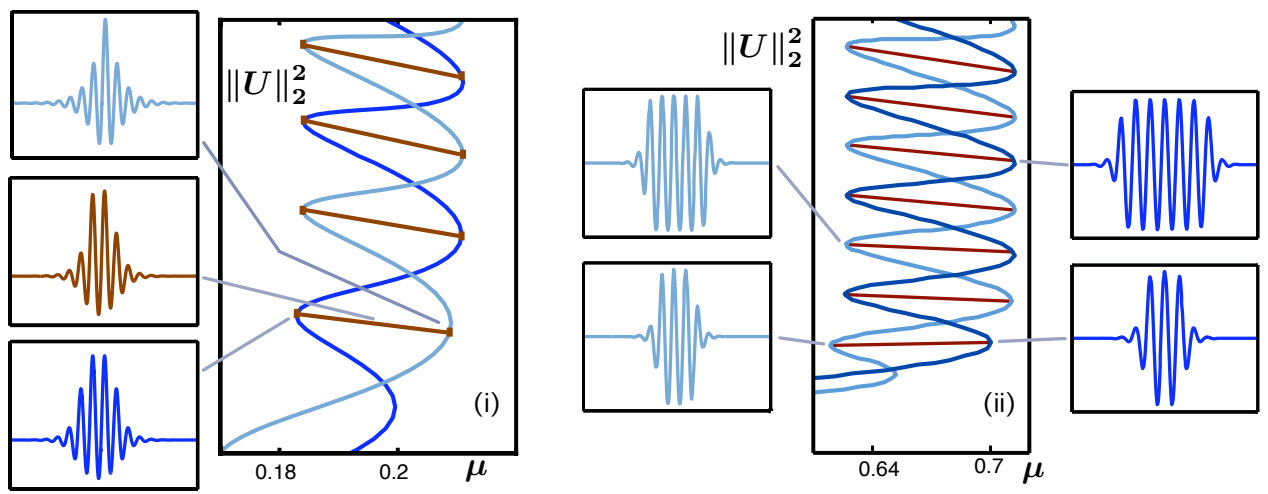

FIG. 1.1. Bifurcation diagrams of localized rolls are plotted in panels (i) and (ii) for the SwiftHohenberg equations (1.1) with $\nu=1.6$ and (1.2) with $\nu=2$, respectively. The insets show localized rolls $U(x)$ as functions of $x$. Solutions on the vertical snaking branches are symmetric under the reflection $x \mapsto-x$, and the $\mathbb{Z}_{2}$-action $U \mapsto-U$ for (1.2), while solutions on the horizontal ladder branches are asymmetric.

The pulses associated with the snaking branches are symmetric with respect to the reflection $x \mapsto-x$. Recently, [5, 4] found numerically that the two snaking branches discussed above are connected by ladder branches associated with asymmetric pulses as shown in Figure 1.1(i). The asymmetric pulses bifurcate from symmetric pulses via pitchfork bifurcations.

Snaking has also been observed in the cubic-quintic Swift-Hohenberg equation

$$
U_{t}=-\left(1+\partial_{x}^{2}\right)^{2} U-\mu U+\nu U^{3}-U^{5}
$$

see, for instance, $[5,4,15,16,24,32,33,34]$. Equation (1.2) is equivariant under the $\mathbb{Z}_{2}$-action $U \mapsto-U$, and we can expect to find both even and odd localized states. This is indeed the case, and a summary of the resulting snaking observed in the references mentioned above is reproduced in Figure 1.1(ii).

Planar steady states that are localized modulations in one or two spatial directions of an underlying domain-covering structure such as hexagons or rolls can also exhibit snaking, and we refer to $[1,23]$ for recent computations for the planar Swift-Hohenberg equation

$$
U_{t}=-(1+\Delta)^{2} U-\mu U+\nu U^{2}-U^{3}, \quad x \in \mathbb{R}^{2} .
$$

To illustrate this phenomenon, we reproduce a computation from [23] in Figure 1.2. Slanted snaking, where the snaking curve is not vertical but slanted to one side, has also been observed recently in $[9,12]$.

On a geometric level, snaking of symmetric localized 1D roll structures is well understood, and we recall briefly the key arguments from $[29,44,8]$. To this end, we write the steady-state equation

$$
-\left(1+\partial_{x}^{2}\right)^{2} U-\mu U+\nu U^{2}-U^{3}=0
$$

associated with (1.1) as the first-order system

$$
u_{x}=f(u, \mu), \quad u=\left(U, U_{x}, U_{x x}, U_{x x x}\right) \in \mathbb{R}^{4},
$$



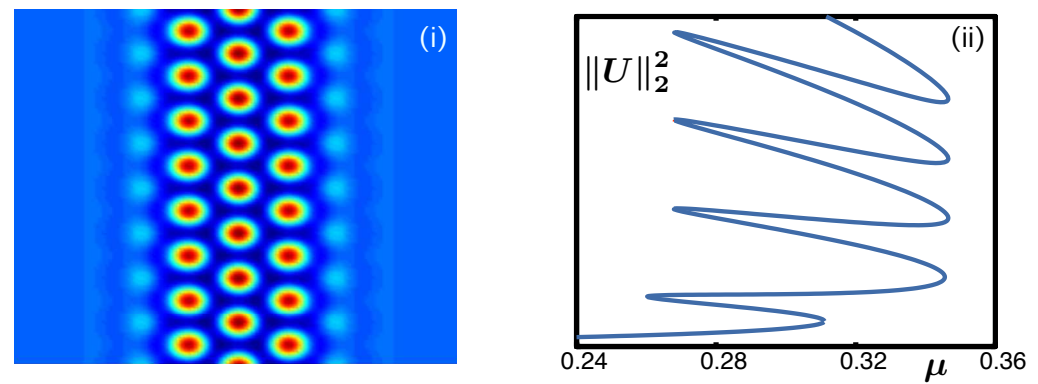

Fig. 1.2. Panel (i) shows a contour plot of a stationary solution of the planar Swift-Hohenberg equation (1.3) for $(\mu, \nu)=(0.31,1.6)$. The solution is localized in the horizontal direction and periodic in the vertical direction. Panel (ii) shows the corresponding snaking curve. Moving up on the snaking curve causes the hexagonal region of the solution in panel (i) to expand in the horizontal direction. Reproduced from [23].

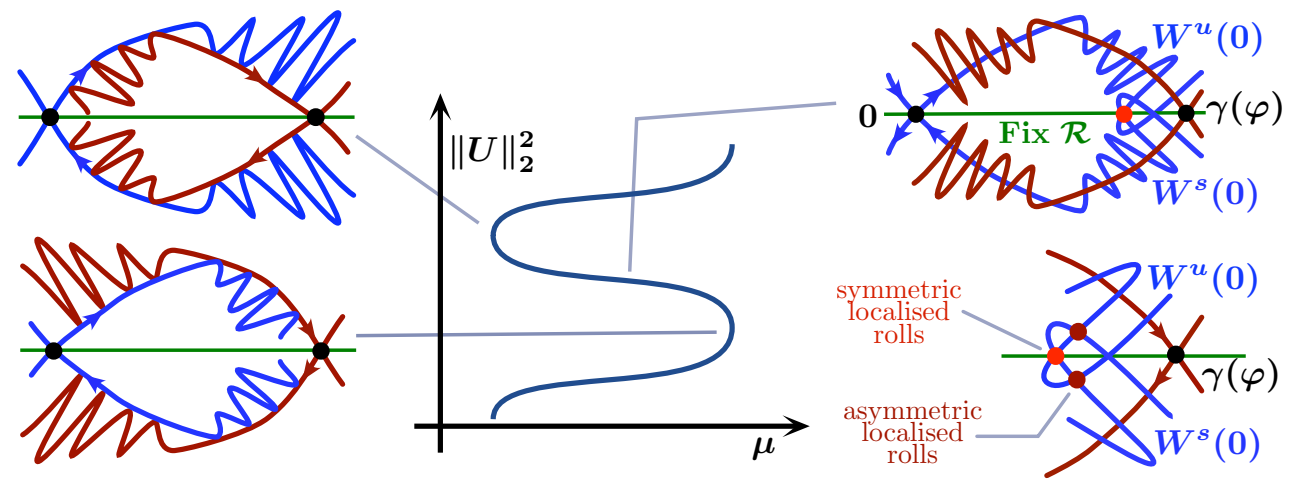

FIG. 1.3. Localized rolls can be found as intersections of the stable and unstable manifolds of $u=0$ near periodic orbits $\gamma$ with $\gamma(\varphi) \in \mathrm{Fix} \mathcal{R}$. These intersections appear and disappear in fold bifurcations. As indicated here schematically, heteroclinic connections between $u=0$ and rolls $\gamma$ organize the overall dynamics, and their fold bifurcations determine the asymptotic limits of the folds associated with localized rolls.

where we chose to omit the dependence on $\nu$, which we keep fixed. Equation (1.4) has several interesting features. First, it is conservative as it admits the first integral

$$
\mathcal{H}(u, \mu)=u_{2} u_{4}-\frac{u_{3}^{2}}{2}+u_{2}^{2}+\frac{(1+\mu) u_{1}^{2}}{2}-\frac{\nu u_{1}^{3}}{3}+\frac{u_{1}^{4}}{4},
$$

whose value does not change along solutions $u(x)$ of (1.4). Furthermore, the $\mathbb{Z}_{2^{-}}$ symmetry $x \mapsto-x$ of the Swift-Hohenberg equation implies that (1.4) is reversible with reverser $\mathcal{R} u=\left(u_{1},-u_{2}, u_{3},-u_{4}\right)$ which, by definition, means that $u(x)$ satisfies (1.4) if and only if $\mathcal{R} u(-x)$ does. We say that a solution $u(x)$ is symmetric if $u(x)=$ $\mathcal{R} u(-x)$ for all $x$, which is equivalent to $u(0) \in \operatorname{Fix} \mathcal{R}$, the fixed-point space of $\mathcal{R}$, and guarantees that the associated solution $U(x)$ is even. Rolls correspond to symmetric periodic orbits of (1.4), and pulses correspond to orbits homoclinic to the origin that pass close to the periodic orbits.

The first integral allows us to restrict (1.4) to the three-dimensional zero energy level set $\mathcal{H}^{-1}(0)$, which is invariant under the flow associated with (1.4). Choosing an appropriate Poincaré section, we can further reduce the dynamics of (1.4) near a periodic orbit $\gamma(x)$ with zero energy to a two-dimensional map. A schematic picture of 

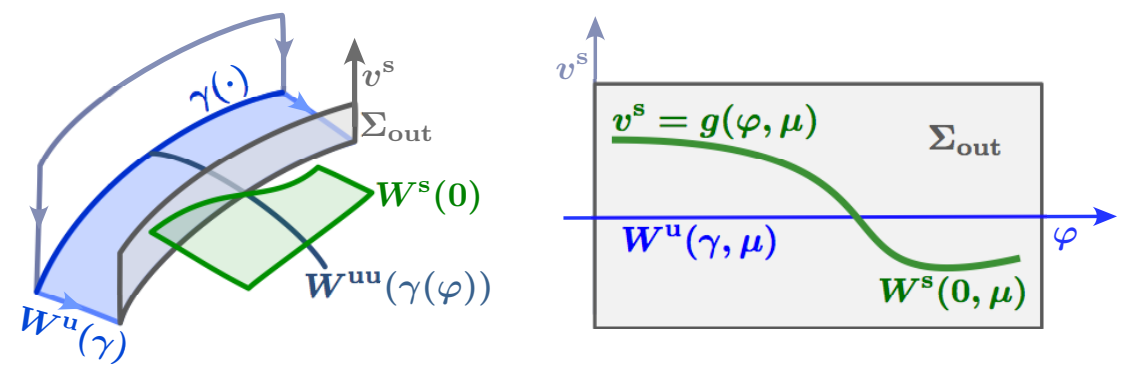

FIG. 1.4. Intersections of $W^{\mathrm{s}}(0, \mu)$ with the strong unstable fiber $W^{\mathrm{uu}}(\gamma(\varphi), \mu)$ of the point $\gamma(\varphi)$ on the periodic orbit $\gamma(\cdot)$ are measured in the section $\Sigma_{\text {out }}$. We assume that the stable manifold $W^{\mathrm{s}}(0, \mu)$ is, locally near each heteroclinic orbit, a graph over the unstable manifold $W^{\mathrm{u}}(\gamma, \mu)$.

the expected dynamics of this map is shown in Figure 1.3. The assumption underlying Figure 1.3 is that heteroclinic orbits that connect the origin to the periodic orbit appear and disappear in fold bifurcations as the parameter $\mu$ is varied. Once these heteroclinic orbits are present, homoclinic orbits to the origin that pass near the periodic orbit appear also. If we plot their $L^{2}$-norm against the parameter $\mu$, we obtain the characteristic snaking curve shown in the center panel of Figure 1.3, and we refer to $[44,8]$ and $[23, \S 2.1]$ for geometric arguments that show why the bifurcation branch has this shape. Figure 1.3 shows that the folds of each homoclinic snaking curve line up with the folds of the heteroclinic orbits. The reduction outlined above can be carried out near each intersection point of $\gamma(x)$ with Fix $\mathcal{R}$, and, since symmetric periodic orbits intersect Fix $\mathcal{R}$ precisely twice [42], we obtain two distinct snaking curves of symmetric homoclinic orbits, in agreement with Figure 1.1.

The goal of this paper is to formulate a set of hypotheses about the connecting orbits between the trivial state and rolls that guarantee snaking. Our approach captures not only the bifurcation curves of symmetric localized structures but also of asymmetric solutions. In the remainder of the introduction, we outline the key assumptions we shall make and the conclusions we can draw from them; we refer to $\S 2$ for precise statements.

As indicated in Figure 1.3, we expect that the overall dynamics of snaking is organized by heteroclinic connections between the trivial state and rolls. We consider a general reversible conservative system of the form (1.4) and assume that it has a periodic orbit $\gamma(x)$ of period $2 \pi$, say, with zero energy ${ }^{1}$ for each $\mu$. We are interested in describing heteroclinic orbits that connect the trivial equilibrium to the periodic orbit $\gamma$. To do so, we choose a section $\Sigma_{\text {out }}$ as indicated in Figure 1.4 and consider intersections of the stable manifold $W^{\mathrm{s}}(0, \mu)$ of $u=0$ with the strong unstable fiber $W^{\text {uu }}(\gamma(\varphi), \mu)$ of the point $\gamma(\varphi)$ on the periodic orbit $\gamma$ in $\Sigma_{\text {out }}$. Such intersections are encoded in the set

$$
\Gamma:=\left\{(\varphi, \mu) \in S^{1} \times \mathbb{R}: W^{\mathrm{s}}(0, \mu) \cap W^{\mathrm{uu}}(\gamma(\varphi), \mu) \cap \Sigma_{\text {out }} \neq \emptyset\right\}, \quad S^{1}=[0,2 \pi] / \sim,
$$

and Figure 1.4 illustrates the additional assumption that the stable manifold $W^{\mathrm{s}}(0, \mu)$ in the section $\Sigma_{\text {out }}$ is the graph of a smooth function $g(\varphi, \mu)$, locally near each heteroclinic orbit; we refer to [13] for the numerical verification of this scenario for an equation of complex Ginzburg-Landau type with constant forcing added. For the

\footnotetext{
${ }^{1}$ The orbit, and its period, may depend on $\mu$ but we ignore this in our outline
} 

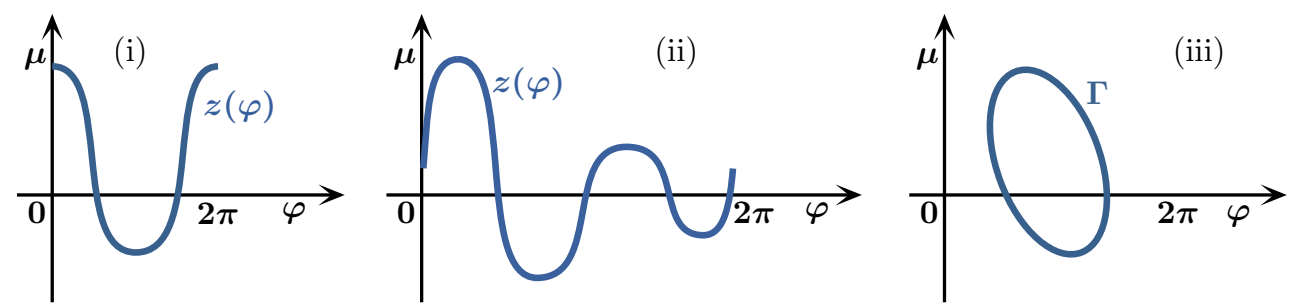

FIG. 1.5. Three example sets $\Gamma$ are shown. The set $\Gamma$ shown in panel (iii) is not a graph and corresponds to an isola of heteroclinic orbits.
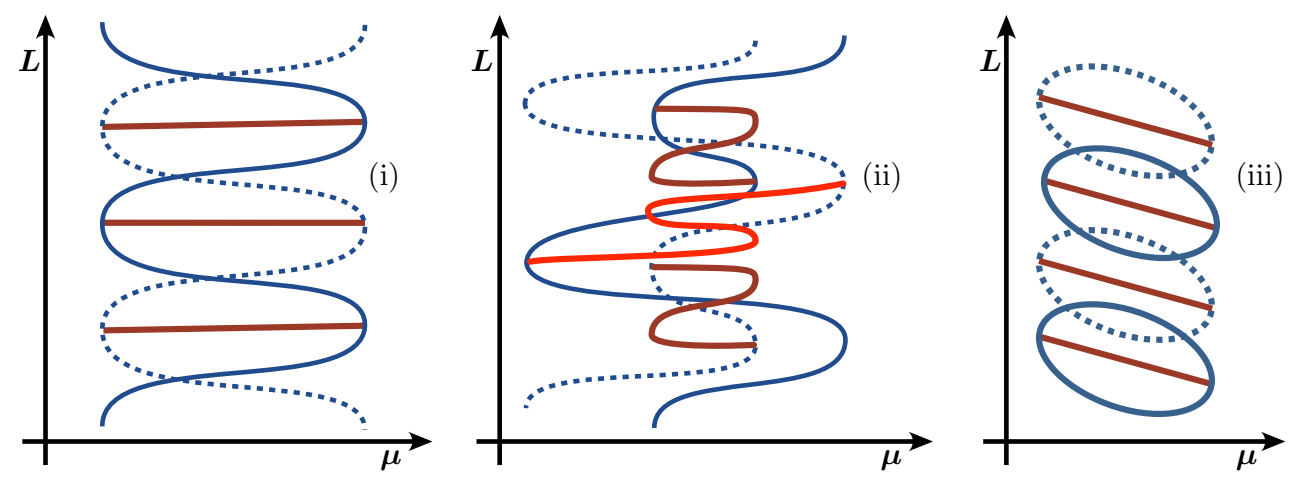

FIG. 1.6. The bifurcation curves of symmetric and asymmetric localized rolls associated with the sets $\Gamma$ from Figure 1.5 are plotted. The vertical axis $L$ measures the "time" $x$ spent by the homoclinic orbit in the vicinity of the periodic orbit $\gamma$ and is therefore roughly equivalent to the $L^{2}$-norm of the corresponding localized state. The vertical snaking curves in panels (i) and (ii) and the sequence of ellipsoids in panel (iii) correspond to symmetric pulses, while the horizontal and $Z$-like branches correspond to asymmetric pulses. Note that the dashed/solid curves in panels (i) and (ii) correspond to the dark/light snaking curves in Figure 1.1.

sake of clarity, we focus on the case where $\Gamma$ is the graph of a function $z$,

$$
\Gamma=\left\{(\varphi, \mu) \in S^{1} \times \mathbb{R}: \mu=z(\varphi)\right\},
$$

and examples are shown in Figure 1.5(i)-(ii). The set $\Gamma$ drawn in Figure 1.5(iii) is not a graph, and we shall outline in $\S 6.1$ how our results can be adapted to cover this situation.

Our main results assert that the complete bifurcation diagram of symmetric and asymmetric pulses can be drawn using only the function $z$, and we plot in Figure 1.6 the diagrams corresponding to the functions given in Figure 1.5. We now outline how the function $z$ determines the bifurcation diagram. We may assume that $\gamma\left(\varphi_{0}\right) \in$ Fix $\mathcal{R}$ if and only if $\varphi_{0} \in\{0, \pi\}$. Symmetric pulses that spend time ${ }^{2} 2 L$ near the periodic orbit $\gamma$ exist if and only if

$$
\mu=\mu_{*}\left(L, \varphi_{0}\right)=z\left(L+\varphi_{0}\right)+\mathrm{O}\left(\mathrm{e}^{-\eta L}\right), \quad L \gg 1
$$

for some $\eta>0$, where $\varphi_{0} \in\{0, \pi\}$. Furthermore, the bifurcation curves of asymmetric pulses that spend time $2 L$ in the vicinity of the periodic orbit $\gamma$ are exponentially close

\footnotetext{
2 "Time" refers to the evolution variable $x$ of (1.4) and therefore corresponds to spatial extent of the resulting patterns
} 


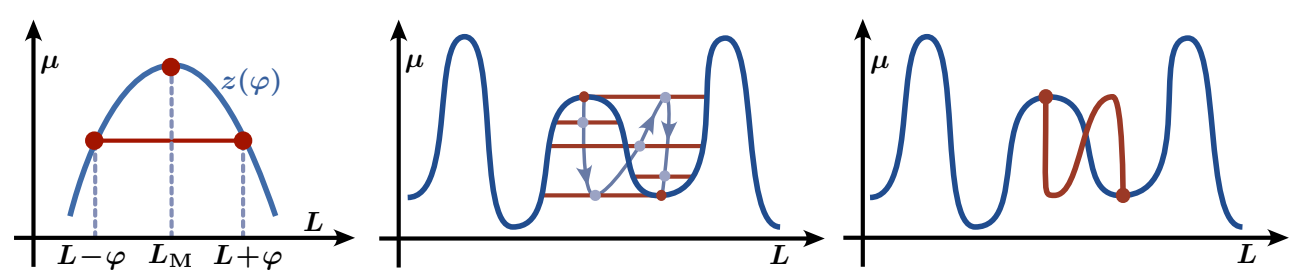

FIG. 1.7. Solutions $(L, \varphi)$ of (1.6) can be found graphically by finding two intersection points of a fixed horizontal line with the graph of $z$ whose abscissas give $L \pm \varphi$. Continuing these solutions by moving the horizontal line up and down generates the entire bifurcation branch. An example is given in the center panel, and the resulting bifurcation curve $(L, \mu)$ is plotted in the right panel. If $\varphi$ reaches $\pi$, the corresponding branch terminates at the second snaking curve.

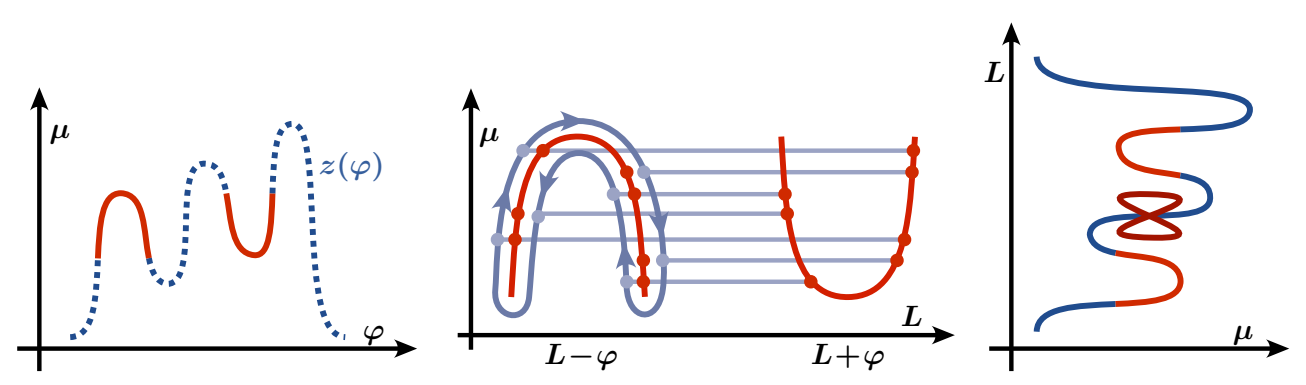

FIG. 1.8. The function $z$ plotted in the left panel generates the figure-eight like isola of asymmetric pulses shown in the right panel. The center panel explains how the positions of $L \pm \varphi$ that satisfy $z(L-\varphi)=z(L+\varphi)$ can be computed using the procedure from Figure 1.7: the solution $(L, \varphi)$ is unique and periodic, and therefore generates an isola.

in $L$ to the set

$$
\left\{(L, \mu)=(L, z(L+\varphi)): z(L+\varphi)=z(L-\varphi) \text { for some } \varphi \in S^{1}\right\} .
$$

This set is determined by the solutions $(L, \varphi) \in \mathbb{R}^{+} \times S^{1}$ of

$$
z(L+\varphi)=z(L-\varphi),
$$

which can be found graphically as outlined in Figure 1.7. Using Figure 1.7, it is not difficult to obtain the diagrams given in Figure 1.6 for the sets $\Gamma$ given in Figure 1.5. We will also prove that the asymmetric branches that emerge from maxima of $z$ will terminate at minima. All asymmetric branches that bifurcate from the symmetric branch (1.5) with $\varphi_{0}=0$ terminate at the same branch except for the branch emerging from the global maximum of $z$, which will connect to the symmetric branch (1.5) with $\varphi_{0}=\pi$, and vice versa. The start and end points of the asymmetric branches that emerge from and terminate at symmetric branches correspond to pitchfork bifurcations. Interestingly, isolas of asymmetric solutions are also possible provided the function $z$ has more than two maxima, and an example is shown in Figure 1.8. We emphasize that (1.6) is $2 \pi$-periodic in $L$ so that each solution curve of (1.6) gives an infinite sequence of branches of asymmetric pulses that are spaced at distances $2 \pi$ along the $L$-axis as indicated in panels (i) and (iii) of Figure 1.6.

A practical implication of these results is that all branches of asymmetric pulses, including isolas, can be predicted once the snaking curves of the symmetric states have been computed. Indeed, Figure 1.7 illustrates how these branches can be found 
from the function $z$, while (1.5) establishes that the branch $\mu_{*}\left(L, \varphi_{0}\right)$ of symmetric pulses is exponentially close to the graph $\mu=z\left(L+\varphi_{0}\right)$ of $z$, so that we can reliably determine $z$ through numerical computations of the branch $\mu_{*}\left(L, \varphi_{0}\right)$ of symmetric pulses. This is of interest as symmetric pulses are typically quite easy to compute since they, in contrast to asymmetric pulses, lie on one or two single branches.

Our choice of example graphs in Figures 1.5 and 1.6 is motivated by those encountered in numerical computations. The bifurcation diagrams in Figure 1.6(i) correspond to those reproduced in Figure 1.1, while bifurcation diagrams similar to Figure 1.6(ii) have recently been computed in [1]. A sequence of isolas of pulses, similar to the one shown in Figure 1.6(iii), has been found in [3, Figure 24] for (1.1), which suggests that fronts are present in this situation whose bifurcation structure resembles Figure 1.5(iii).

In the preceding discussion, we focused on conservative reversible differential equations. We wish to emphasize that reversibility is the only feature essential for snaking to occur. In particular, systems that are reversible, but not conservative, will also exhibit snaking, and we consider this situation in $\S 6.4$. The existence of a conserved quantity guarantees that the bifurcating asymmetric pulses are standing waves: if the system is not conservative, these pulses will travel with a velocity close to zero. We also remark that our results can be extended to planar patterns that are localized in one spatial variable and periodic in the second one: this will be discussed further in $\S 6.2$.

We now discuss related works. Homoclinic orbits that bifurcate in reversible systems from heteroclinic cycles between equilibria have been investigated earlier in [19]. Of relevance for the snaking behaviour discussed here are homoclinic orbits that bifurcate from heteroclinic cycles connecting equilibria to periodic orbits: their geometry was elucidated in $[29,44,8]$ and detailed numerical studies were carried out, for instance, in $[44,3,5,4]$. Analytical gluing results for heteroclinic cycles involving periodic orbits have, to our knowledge, been given only in the context of generic systems without reversibility. In [30], for instance, heteroclinic cycles of codimension one and two were studied, and the role played by the winding number of the stable manifold $W^{\mathrm{s}}(0)$ with respect to the periodic orbit for obtaining global solution branches was illuminated. The proofs given in [30] utilized Lin's method, while we shall transform our system near the periodic orbit into Fenichel's normal form and use reversible Shilnikov variables for the resulting system. For the Swift-Hohenberg equation (1.1), the existence of fronts and of symmetric and asymmetric pulses was recently investigated in $[21,7]$ near the onset to rolls, and more specifically near the codimension-two point $(\mu, \nu)=(0, \sqrt{27 / 38})$, using formal asymptotics beyond all orders. We complement these results by proving how fronts between rolls and the trivial state can be glued together to produce localized states. Our analysis is not limited to the parameter region near onset, but, in contrast to [21,7], it is not capable of establishing the existence of fronts in the first place. We compare our results in more detail in $\S 7$ with those obtained in $[21,7]$.

The rest of the paper is organized as follows. In $\S 2$, we state our hypotheses and the main results for systems in $\mathbb{R}^{4}$. We introduce reversible Shilnikov variables in $\S 3$, and the proofs of the main results for symmetric and asymmetric pulses are given in $\S 4$ and $\S 5$, respectively. Various extensions, for instance to isolas and to higherdimensional systems including $2 \mathrm{D}$ patterns, are outlined and proved in $\S 6$. We end in $\S 7$ with a discussion of the Swift-Hohenberg equation and the stability of symmetric and asymmetric localized states with respect to the underlying partial differential 
equation (PDE).

2. Main results. We consider ordinary differential equations

$$
u_{x}=f(u, \mu), \quad u \in \mathbb{R}^{4}, \quad \mu \in \mathbb{R}
$$

in $\mathbb{R}^{4}$, where $f$ is assumed to be a smooth function, and remark that extensions to higher-dimensional systems are discussed in $\S 6.2$. We are interested in reversible systems and therefore assume the following.

Hypothesis 1 . There exists a linear map $\mathcal{R}: \mathbb{R}^{4} \rightarrow \mathbb{R}^{4}$ with $\mathcal{R}^{2}=1$ and $\operatorname{dim} \operatorname{Fix} \mathcal{R}=2$ so that $f(\mathcal{R} u, \mu)=-\mathcal{R} f(u, \mu)$ for all $(u, \mu)$.

From the reversibility hypothesis 1 , we infer that $\mathcal{R} u(-x)$ satisfies $(2.1)$ whenever $u(x)$ does. We say that a solution $u(x)$ is symmetric or reversible if $u(0) \in \operatorname{Fix} \mathcal{R}$, which implies that $u(x)=\mathcal{R} u(-x)$ for all $x$.

Next, we assume that (2.1) is conservative which, by definition, means that it has a first integral $\mathcal{H}$, which we assume to be invariant under the reverser $\mathcal{R}$. This assumption is not essential for our analysis, and we refer to $\S 6.4$ for extensions to systems that are reversible but not conservative.

Hypothesis 2. There exists a smooth function $\mathcal{H}: \mathbb{R}^{4} \times \mathbb{R} \rightarrow \mathbb{R}$ with $\mathcal{H}(\mathcal{R} u, \mu)=$ $\mathcal{H}(u, \mu)$ and $\left\langle\nabla_{u} \mathcal{H}(u, \mu), f(u, \mu)\right\rangle=0$ for all $(u, \mu)$. We normalize $\mathcal{H}$ so that $\mathcal{H}(0, \mu)=$ 0 for all $\mu$.

We can now state our assumptions on the specific solutions of (2.1) we are interested in.

HYPOTHESIS 3. The origin $u=0$ is a hyperbolic equilibrium of (2.1). More precisely, we assume that $f(0, \mu)=0$ for all $\mu$ and that $f_{u}(0, \mu)$ has two eigenvalues with strictly negative real part and another two eigenvalues with strictly positive real part.

Hypothesis 1 implies that the spectrum of $f_{u}(0, \mu)$ is invariant under multiplication by -1 . Next, we require that (2.1) has, for each $\mu$ in an appropriate interval, a symmetric periodic orbit in the zero energy level set. Throughout, we reserve the term "periodic orbit" for solutions with nonzero minimal period.

НуротнеSIS 4. We assume that there is a closed interval $J \subset \mathbb{R}$ with nonempty interior $\stackrel{\circ}{J}$ so that (2.1) has, for each $\mu \in J$, a periodic orbit $\gamma(x, \mu)$ with minimal period $\ell(\mu)$ which satisfies:

(i) The family $\gamma(x, \mu)$ depends smoothly on $\mu \in J$.

(ii) $\gamma(x, \mu)$ is symmetric with $\gamma(0, \mu) \in$ Fix $\mathcal{R}$ for all $\mu \in J$.

(iii) $\gamma(x, \mu)$ has zero energy: for each $\mu \in J$, we have $\mathcal{H}(\gamma(x, \mu), \mu)=0$ and $\mathcal{H}_{u}(\gamma(x, \mu), \mu) \neq 0$ for one, and hence all, $x$.

(iv) $\gamma(x, \mu)$ has two positive nontrivial Floquet multipliers $\mathrm{e}^{ \pm 2 \pi \alpha(\mu)}$ with $\alpha(\mu)>0$ for all $\mu \in J$.

Rescaling time if necessary, we can, without loss of generality, assume that the minimal periods $\ell(\mu)$ are all equal to $2 \pi$. In particular, [42] implies that $\gamma(\pi, \mu) \in$ $\operatorname{Fix}(\mathcal{R})$. We will always identify $S^{1}=[0,2 \pi] / \sim$.

Some of our results change if (2.1) has additional symmetries. To account for such systems, we shall sometimes assume that (2.1) respects the reflection symmetry $\kappa: u \mapsto-u$. Consider the variational equation

$$
v_{x}=f_{u}(\gamma(x, \mu), \mu) v
$$

\footnotetext{
${ }^{3}$ The particular form of the Floquet exponents facilitates a rescaling of the period of $\gamma$ to $2 \pi$
} 
about $\gamma(x, \mu)$ which, due to Hypothesis 4(iv), has two nontrivial solutions $v^{\mathrm{s}}$ and $v^{\mathrm{u}}$ of the form

$$
v^{\mathrm{s}}(x)=\mathrm{e}^{-\alpha(\mu) x} p^{\mathrm{s}}(x, \mu), \quad v^{\mathrm{u}}(x)=\mathrm{e}^{\alpha(\mu) x} p^{\mathrm{u}}(x, \mu),
$$

where $p^{j}(x, \mu)$ are $2 \pi$-periodic in $x$. We shall normalize these functions by requiring that

$$
p^{\mathrm{u}}(x, \mu)=\mathcal{R} p^{\mathrm{s}}(-x, \mu)
$$

for all $x$ and $\mu \in J$.

HyPOTHESIS 5. The nonlinearity $f$ is odd in $u$ so that $f(-u, \mu)=-f(u, \mu)$ for all $(u, \mu)$. Furthermore, we assume that the orbit $\gamma(\cdot, \mu)$ is invariant under $\kappa: u \mapsto-u$ and that $p^{\mathrm{s}}(\pi, \mu)=\kappa p^{\mathrm{s}}(0, \mu)$ for all $\mu \in J$.

Hypothesis 5 implies that $\kappa \mathcal{R}=-\mathcal{R}$ is a second reverser of (2.1). Furthermore, the first part of Hypothesis 5 implies that the flow $\Phi_{x}(u)$ of $(2.1)$ satisfies $\kappa \Phi_{x}(u)=$ $\Phi_{x}(\kappa u)$, and therefore $\mathrm{D}_{x}(u)=\mathrm{D} \Phi_{x}(-u)$, for all $x$ and $u$. If the orbit $\gamma(\cdot, \mu)$ is invariant under $\kappa$, then necessarily $\kappa \gamma(x, \mu)=\gamma(x+\pi, \mu)$ for all $x$. In particular, we have $\mathrm{D} \Phi_{\pi}(\gamma(0, \mu))=\mathrm{D} \Phi_{\pi}(\gamma(\pi, \mu))$, which implies that $p^{\mathrm{s}}(\pi, \mu)= \pm p^{\mathrm{s}}(0, \mu)$. The second part of Hypothesis 5 assumes that the negative sign occurs in our system so that, in fact, $\kappa p^{\mathrm{s}}(x, \mu)=p^{\mathrm{s}}(x+\pi, \mu)$ for all $x$.

Lemma 2.1. Assume that Hypotheses 1-4 are met, then there exist a $\delta>0, a$ smooth reversible change of coordinates near $\gamma(\cdot, \mu)$, and smooth real-valued functions $h^{\mathrm{c}}, h_{j}^{\mathrm{s}}$ and $h_{j}^{\mathrm{u}}$ for $j=1,2$ so that (2.1) restricted to the zero energy level set is for all $\mu \in J$ of the form

$$
\begin{aligned}
& v_{x}^{\mathrm{c}}=1+h^{\mathrm{c}}(v, \mu) v^{\mathrm{s}} v^{\mathrm{u}}, \\
& v_{x}^{\mathrm{s}}=-\left[\alpha(\mu)+h_{1}^{\mathrm{s}}(v, \mu) v^{\mathrm{s}}+h_{2}^{\mathrm{s}}(v, \mu) v^{\mathrm{u}}\right] v^{\mathrm{s}}, \\
& v_{x}^{\mathrm{u}}=\left[\alpha(\mu)+h_{1}^{\mathrm{u}}(v, \mu) v^{\mathrm{s}}+h_{2}^{\mathrm{u}}(v, \mu) v^{\mathrm{u}}\right] v^{\mathrm{u}},
\end{aligned}
$$

where $v=\left(v^{\mathrm{c}}, v^{\mathrm{s}}, v^{\mathrm{u}}\right) \in \mathcal{V}:=S^{1} \times I \times I$ and $I=[-\delta, \delta]$. The reverser $\mathcal{R}$ and the symmetry $\kappa$ (if Hypothesis 5 is met) act on $v$ via

$$
\mathcal{R}\left(v^{\mathrm{c}}, v^{\mathrm{s}}, v^{\mathrm{u}}\right)=\left(-v^{\mathrm{c}}, v^{\mathrm{u}}, v^{\mathrm{s}}\right), \quad \kappa\left(v^{\mathrm{c}}, v^{\mathrm{s}}, v^{\mathrm{u}}\right)=\left(v^{\mathrm{c}}+\pi, v^{\mathrm{s}}, v^{\mathrm{u}}\right) .
$$

We shall prove Lemma 2.1 in $\S 3$. Note that $v^{\mathrm{s}}=0$ corresponds to the unstable manifold of the periodic orbit $\gamma(\cdot, \mu)$ and its strong unstable fibers $W^{\text {uu }}(\gamma(\varphi, \mu), \mu)$ are given by further fixing $\varphi$. As illustrated in Figure 1.4, we define the sections

$$
\Sigma_{\text {in }}=S^{1} \times\left\{v^{\mathrm{s}}=\delta\right\} \times I, \quad \Sigma_{\text {out }}=S^{1} \times I \times\left\{v^{\mathrm{u}}=\delta\right\} .
$$

We are interested in orbits that connect the periodic orbits $\gamma(x, \mu)$ to the equilibrium $u=0$, and therefore define

$$
\Gamma:=\left\{(\varphi, \mu) \in S^{1} \times J: W^{\mathrm{s}}(0, \mu) \cap W^{\mathrm{uu}}(\gamma(\varphi, \mu), \mu) \cap \Sigma_{\text {out }} \neq \emptyset\right\} .
$$

As in the introduction, we now assume that $\Gamma$ is a graph and refer to $\S 6.1$ for extensions to isolas of fronts.

HyPOTHESIS 6 . The set $\Gamma$ is the graph of a smooth function $z: S^{1} \rightarrow \stackrel{J}{J}$. Furthermore, there exist an open neighbourhood $U_{\Gamma}$ of $\Gamma$ in $S^{1} \times J$, positive constants $\epsilon, b>0$, and a smooth function $g: U_{\Gamma} \rightarrow I$ so that

$$
\begin{aligned}
& \left\{\left(\varphi, v^{\mathrm{s}}, \delta\right) \in W^{\mathrm{s}}(0, \mu) \cap \Sigma_{\text {out }}:\left|v^{\mathrm{s}}\right|<\epsilon,(\varphi, \mu) \in U_{\Gamma}\right\} \\
& =\left\{\left(\varphi, v^{\mathrm{s}}, \delta\right)=(\varphi, g(\varphi, \mu), \delta):(\varphi, \mu) \in U_{\Gamma}\right\}
\end{aligned}
$$


and $\left|g_{\mu}(\varphi, \mu)\right| \geq b>0$ for all $(\varphi, \mu) \in U_{\Gamma}$.

Hypothesis 6 encodes the bifurcation diagram of fronts or heteroclinic orbits that connect the periodic orbits $\gamma$ to $u=0$ through the intersections of the strong unstable fibers $W^{\mathrm{uu}}(\gamma(x, \mu), \mu)$ and the stable manifold $W^{\mathrm{s}}(0, \mu)$; see also Figures 1.4-1.5. Nondegenerate maxima and minima of $z$ correspond to saddle-node bifurcations of fronts, that is, to quadratic tangencies of $W^{\mathrm{s}}(0, \mu)$ and $W^{\mathrm{uu}}(\gamma(x, \mu), \mu)$ at which heteroclinic connections disappear. For our results on asymmetric pulses, we need to assume that the only critical points of $z$ are nondegenerate maxima or minima which occur at different values of $z$.

Hypothesis 7. We assume that $z^{\prime}(\varphi)=0$ implies $z^{\prime \prime}(\varphi) \neq 0$. Furthermore, we assume that $z\left(\varphi_{1}\right)=z\left(\varphi_{2}\right)$ and $z^{\prime}\left(\varphi_{1}\right)=z^{\prime}\left(\varphi_{2}\right)=0$ implies $\varphi_{1}=\varphi_{2}$ modulo $2 \pi$ (modulo $\pi$ if Hypothesis 5 is met).

We define

$$
\Theta:= \begin{cases}\left\{0, \frac{1}{2} \pi\right\} & \text { if Hypothesis } 5 \text { is met } \\ \{0, \pi\} & \text { otherwise. }\end{cases}
$$

We can now state our two main results on the existence of symmetric and asymmetric 1-pulses, which are homoclinic orbits that follow the cycle formed by the heteroclinic orbits from Hypothesis 6 precisely once.

TheOREm 2.2 (Symmetric 1-pulses). Assume that Hypotheses 1-4 and 6-7 are met, then there are constants $L_{*} \gg 1$ and $\eta>0$ so that the following is true: for each $L>L_{*}$, (2.1) has a symmetric homoclinic orbit $u(x)$ for $\mu \in J$ that spends time $2 L$ in the neighbourhood $\mathcal{V}$ of $\gamma(\cdot, \mu)$ if and only if

$$
\mu=\mu_{*}\left(L, \varphi_{0}\right)=z\left(\varphi_{0}+L\right)+\mathrm{O}\left(\mathrm{e}^{-\eta L}\right)
$$

for an appropriate $\varphi_{0} \in \Theta$, and $u(0)$ lies near $\gamma\left(\varphi_{0}, \mu\right)$ in $\operatorname{Fix}(\mathcal{R})$ for $\varphi_{0} \in\{0, \pi\}$.

If Hypothesis 5 is met, too, then $z$ is $\pi$-periodic, the above statements are true for $\varphi_{0} \in\{0, \pi / 2\}$, and $u(0)$ lies near $\gamma(\pi / 2, \mu)$ in $\operatorname{Fix}(-\mathcal{R})$ when $\varphi_{0}=\pi / 2$. Furthermore, symmetric 1-pulses come in pairs: if $u$ is a 1-pulse corresponding to $\varphi_{0}$, then $-u$ is a 1-pulse corresponding to $\varphi_{0}+\pi$.

Lemma 4.1 contains precise expansions of $\mu_{*}$ and the associated fold bifurcations that sharpen the expansion (2.7) given in the preceding theorem. The following theorem makes Figure 1.6 precise: the functions $a_{i}(\cdot, n)=a_{i}(\cdot)+$ h.o.t. describe the vertical sequence of horizontal branches of asymmetric pulses that begin and end at pitchfork bifurcations of symmetric pulses, where $i$ runs from 1 to the number of maxima of $z$, and $n$ parametrizes the infinite vertical sequence; similarly, the functions $\iota_{j}(\cdot, n)=\iota_{j}(\cdot)+$ h.o.t. correspond to an infinite sequence of isolas of asymmetric pulses which, as indicated in Figure 1.8, may also be present. We denote by

$$
\mathcal{S}_{\varphi_{0}}:=\left\{(\mu, L)=\left(\mu_{*}\left(L, \varphi_{0}\right), L\right): L>L_{*}\right\} \subset \mathbb{R}^{2}, \quad \varphi_{0} \in \Theta
$$

the two branches of symmetric 1-pulses described in Theorem 2.2.

Theorem 2.3 (Asymmetric 1-pulses). Assume that Hypotheses 1-4 and 6-7 are met, and denote by $k$ the number of maxima of $z$. There are then an $\eta>0$, natural numbers $n_{*} \gg 1$ and $\tilde{k} \geq 0$, and smooth curves

$$
a_{i}, a_{i}\left(\cdot, n, \varphi_{0}\right), \iota_{j}, \iota_{j}\left(\cdot, n, \varphi_{0}\right):[0,1] \longrightarrow \mathbb{R}^{2}, \quad 1 \leq i \leq k, \quad 1 \leq j \leq \tilde{k}
$$

defined for $n>n_{*}$ and $\varphi_{0} \in \Theta$ so that the following is true for $n>n_{*}$ and $\varphi_{0} \in \Theta$ : 

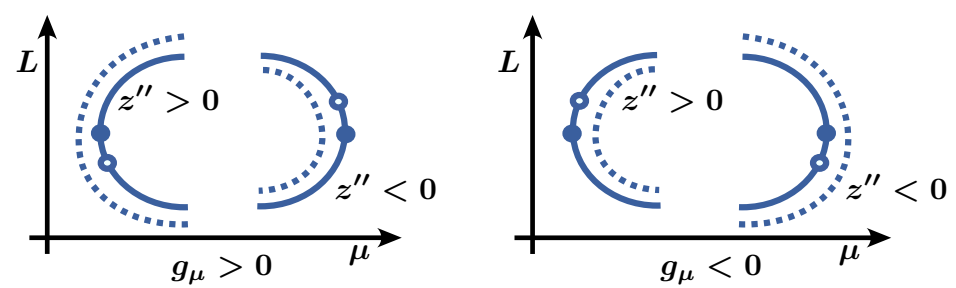

FIG. 2.1. Shown are the offset of the snaking curve of symmetric 1-pulses [solid line] from the limiting curve that corresponds to fronts [dotted line], and the relative positions of saddle-node [filled circles] and pitchfork bifurcations [open circles] along the snaking curve of symmetric 1-pulses; see also Remark 1.

(i) We have

$$
a_{i}\left(s, n, \varphi_{0}\right)=a_{i}(s)+\left(0, \varphi_{0}+2 \pi n\right)+\mathrm{O}\left(\mathrm{e}^{-\eta n}\right), \quad s \in[0,1], 1 \leq i \leq k .
$$

For $1 \leq i<k$, the curves $a_{i}\left(s, n, \varphi_{0}\right)$ begin for $s=0$ in $\mathcal{S}_{\varphi_{0}}$ near a non-global maximum $^{4}$ of $z$ and terminate for $s=1$ in $\mathcal{S}_{\varphi_{0}}$ near a non-global minimum of $z$. The curves $a_{k}\left(s, n, \varphi_{0}\right)$ begin for $s=0$ in $\mathcal{S}_{\varphi_{0}}$ near the global maximum of $z$ and terminate for $s=1$ in $\mathcal{S}_{\varphi_{0}+\pi}$ near the global minimum of $z$.

(ii) We have

$$
\iota_{j}\left(s, n, \varphi_{0}\right)=\iota_{j}(s)+\left(0, \varphi_{0}+2 \pi n\right)+\mathrm{O}\left(\mathrm{e}^{-\eta n}\right), \quad \iota_{j}\left(0, n, \varphi_{0}\right)=\iota_{j}\left(1, n, \varphi_{0}\right)
$$

for $s \in[0,1]$ and $1 \leq j \leq \tilde{k}$.

(iii) Equation (2.1) has an asymmetric homoclinic orbit $u(x)$ for $\mu \in J$ that spends time $2 L$ in the neighbourhood $\mathcal{V}$ of $\gamma(\cdot, \mu)$ if and only if $(\mu, L)$ lies on one of the curves $a_{i}\left(s, n, \varphi_{0}\right)$ with $s \neq 0,1$ or on $\iota_{j}\left(s, n, \varphi_{0}\right)$ for $s \in[0,1]$.

(iv) The start and end points of the curves $a_{i}\left(s, n, \varphi_{0}\right)$ for $s=0,1$ correspond to pitchfork bifurcations of the symmetric 1-pulses described in Theorem 2.2; no other pitchfork bifurcations occur on $\mathcal{S}_{\varphi_{0}}$. The curves $\iota_{j}\left(\cdot, n, \varphi_{0}\right)$ correspond to isolas of asymmetric solutions. Furthermore, the curves $a_{i}(s)$ and $\iota_{j}(s)$ are obtained from solutions $(L, \varphi)$ of (1.6) with $L \in S^{1}$ upon setting $\mu=z(L+\varphi)$. Finally, if Hypothesis 5 is met, then the above statements remain true provided we replace $\mathcal{S}_{\varphi_{0}+\pi}$ in (i) by $\mathcal{S}_{\varphi_{0}+\pi / 2}$. Furthermore, if $u$ is an asymmetric 1-pulse, so are $\mathcal{R} u,-u$, and $-\mathcal{R} u$.

We refer to Lemma 5.1 for expansions of the location of the pitchfork bifurcations that corroborate the summary given in Figure 2.1. Lemma 5.4 shows that isolas are only possible when $k \geq 3$, and an example of a function $z$ with three maxima that leads to isolas is given in Figure 1.8.

3. Reversible Shilnikov variables near a periodic orbit. We begin with the proof of Lemma 2.1 .

Proof. [ of Lemma 2.1.] The desired coordinate change will straighten out the stable and unstable manifolds of the periodic orbit $\gamma(x, \mu)$ inside the zero level set of the energy $\mathcal{H}(\cdot, \mu)$. Recall that the variational equation $v_{x}=f_{u}(\gamma(x, \mu), \mu) v$ around the periodic orbit $\gamma(x, \mu)$ admits the two solutions

$$
v^{\mathrm{s}}(x)=\mathrm{e}^{-\alpha(\mu) x} p^{\mathrm{s}}(x, \mu), \quad v^{\mathrm{u}}(x)=\mathrm{e}^{\alpha(\mu) x} p^{\mathrm{u}}(x, \mu)
$$

${ }^{4}$ That is, near a point $\left(\mu_{*}, L_{*}\right)$ on $\mathcal{S}_{\varphi_{0}}$ given by $(2.7)$ where $z\left(\varphi_{0}+L\right)$ has a non-global maximum at $L=L_{*}$ 
given in $(2.2)$, where $p^{j}(x, \mu)$ is $2 \pi$-periodic in $x$. Thus, the tangent spaces of the strong stable and unstable fibers of $\gamma(x, \mu)$ are spanned by $p^{\mathrm{s}}(x, \mu)$ and $p^{\mathrm{u}}(x, \mu)$, respectively. On the linear level, omitting higher-order corrections to the invariant manifolds of the periodic orbit, this suggests to use the transformation

$$
v=\left(v^{\mathrm{c}}, v^{\mathrm{s}}, v^{\mathrm{u}}\right) \in S^{1} \times I \times I \longmapsto u=\gamma\left(v^{\mathrm{c}}, \mu\right)+v^{\mathrm{s}} p^{\mathrm{s}}\left(v^{\mathrm{c}}, \mu\right)+v^{\mathrm{u}} p^{\mathrm{u}}\left(v^{\mathrm{c}}, \mu\right),
$$

which maps $\left(v^{\mathrm{c}}, 0,0\right)$ onto the periodic orbit, and $\left(v^{\mathrm{c}}, v^{\mathrm{s}}, 0\right)$ and $\left(v^{\mathrm{c}}, 0, v^{\mathrm{u}}\right)$ onto the tangent spaces of the stable and unstable manifolds. We first determine the action of $\mathcal{R}$ on $v$ : the calculation

$$
\begin{array}{r}
\mathcal{R} u=\mathcal{R} \gamma\left(v^{\mathrm{c}}, \mu\right)+v^{\mathrm{s}} \mathcal{R} p^{\mathrm{s}}\left(v^{\mathrm{c}}, \mu\right)+v^{\mathrm{u}} \mathcal{R} p^{\mathrm{u}}\left(v^{\mathrm{c}}, \mu\right) \\
\stackrel{(2.3)}{=} \gamma\left(-v^{\mathrm{c}}, \mu\right)+v^{\mathrm{s}} p^{\mathrm{u}}\left(-v^{\mathrm{c}}, \mu\right)+v^{\mathrm{u}} p^{\mathrm{s}}\left(-v^{\mathrm{c}}, \mu\right)
\end{array}
$$

shows that $\mathcal{R} u$ corresponds to $\mathcal{R} v=\mathcal{R}\left(v^{\mathrm{c}}, v^{\mathrm{s}}, v^{\mathrm{u}}\right)=\left(-v^{\mathrm{c}}, v^{\mathrm{u}}, v^{\mathrm{s}}\right)$, which gives the representation of $\mathcal{R}$ stated in (2.5). Similarly, Hypothesis 5 and the discussion following it imply that

$$
\begin{aligned}
\kappa u & =\kappa \gamma\left(v^{\mathrm{c}}, \mu\right)+v^{\mathrm{s}} \kappa p^{\mathrm{s}}\left(v^{\mathrm{c}}, \mu\right)+v^{\mathrm{u}} \kappa p^{\mathrm{u}}\left(v^{\mathrm{c}}, \mu\right) \\
& =\gamma\left(v^{\mathrm{c}}+\pi, \mu\right)+v^{\mathrm{s}} p^{\mathrm{s}}\left(v^{\mathrm{c}}+\pi, \mu\right)+v^{\mathrm{u}} p^{\mathrm{u}}\left(v^{\mathrm{c}}+\pi, \mu\right),
\end{aligned}
$$

which shows that $\kappa u$ corresponds to $\kappa v=\left(v^{\mathrm{c}}+\pi, v^{\mathrm{s}}, v^{\mathrm{u}}\right)$ as asserted in (2.5). The transformation (3.1) therefore shows that the zero energy level set near $\gamma(\cdot, \mu)$ is diffeomorphic to $S^{1} \times I \times I$. Amending this transformation appropriately so that $\left(v^{\mathrm{c}}, v^{\mathrm{s}}, 0\right)$ and $\left(v^{\mathrm{c}}, 0, v^{\mathrm{u}}\right)$ parametrize the strong stable and unstable fibers of $\gamma\left(v^{\mathrm{c}}, \mu\right)$ on the periodic orbit in a reversible and equivariant fashion, and subsequently using the transformation $v \mapsto u$ to straighten out the invariant stable and unstable manifolds of the periodic orbit and its stable and unstable fibers transforms (2.1) into (2.4) locally near the periodic orbit [11].

We now solve (2.4) near the periodic orbit.

Lemma 3.1. There exist positive constants $L_{0}$ and $\eta$ so that the following is true for all $L>L_{0}$ and $\varphi \in S^{1}$ : there is a unique solution $v(x)$, also referred to as $v(x, \varphi)$, of (2.4), defined for $x \in[-L, L]$, so that

$$
v(-L) \in \Sigma_{\text {in }}, \quad v(L) \in \Sigma_{\text {out }}, \quad v^{\mathrm{c}}(0)=\varphi, \quad v(x) \in \mathcal{V} \quad \forall x \in[-L, L] .
$$

Furthermore, we have

$$
\begin{aligned}
v(-L) & =\left(\varphi-L+\mathrm{O}\left(\mathrm{e}^{-\eta L}\right), \delta, \delta \mathrm{e}^{-2 \alpha(\mu) L}\left(1+\mathrm{O}\left(\mathrm{e}^{-\eta L}\right)\right)\right), \\
v(L) & =\left(\varphi+L+\mathrm{O}\left(\mathrm{e}^{-\eta L}\right), \delta \mathrm{e}^{-2 \alpha(\mu) L}\left(1+\mathrm{O}\left(\mathrm{e}^{-\eta L}\right)\right), \delta\right), \\
v(0) & =\left(\varphi, \delta \mathrm{e}^{-\alpha(\mu) L}\left(1+\mathrm{O}\left(\mathrm{e}^{-\eta L}\right)\right), \delta \mathrm{e}^{-\alpha(\mu) L}\left(1+\mathrm{O}\left(\mathrm{e}^{-\eta L}\right)\right)\right) .
\end{aligned}
$$

The solution $v(x)$ is smooth in $(\varphi, \mu, L)$, and the error estimates in (3.2) can be differentiated. Furthermore, we have

$$
v(x,-\varphi)=\mathcal{R} v(-x, \varphi), \quad \varphi \in S^{1},|x| \leq L
$$

and, if Hypothesis 5 holds,

$$
v(x, \pi+\varphi)=\kappa v(x, \varphi), \quad \varphi \in S^{1},|x| \leq L,
$$


where $\mathcal{R}$ and $\kappa$ act according to (2.5). In particular, the solution $v(x, \varphi)$ is $\mathcal{R}$ reversible, with $v(0) \in \operatorname{Fix}(\mathcal{R})$, if and only if $\varphi=0, \pi$, and $\kappa \mathcal{R}$-reversible, with $v(0) \in \operatorname{Fix}(\kappa \mathcal{R})$, if and only if $\varphi=\pi / 2,3 \pi / 2$.

Proof. Existence and uniqueness follow, for instance, from [22, Theorem 4 and $\S 5]$ or [40, Theorem 2.1]. The expansion for $v(x)$ follows from [40, Theorem 2.1], while the expansions for $v^{\mathrm{s}}(x)$ and $v^{\mathrm{u}}(x)$ follow then as in [22, §5] or [35]. The claims about reversibility follow from the expression for $v^{\mathrm{c}}(0)$ together with the uniqueness of the solution considered in the lemma. Note that (2.5) implies that $\mathcal{R} \Sigma_{\text {in }}=\Sigma_{\text {out }}$ and that the elements on the periodic orbits fixed by $\mathcal{R}$ and $\kappa \mathcal{R}$ are $\varphi=0, \pi$ and $\varphi=\pi / 2,3 \pi / 2$, respectively. $\square$

In the proofs given in $\S 4$ and $\S 5$, we shall denote different positive exponential rates $\eta$ that appear in estimates of remainder terms by the same constant, as they are bounded from below by the same positive constant.

Next, we consider the intersection of $W^{\mathrm{u}}(0, \mu)$ with $\Sigma_{\text {in }}$.

Lemma 3.2. We have $v=\left(\varphi, \delta, v^{\mathrm{u}}\right) \in W^{\mathrm{u}}(0, \mu)$ if and only if $\mathcal{R} v=\left(-\varphi, v^{\mathrm{u}}, \delta\right) \in$ $W^{\mathrm{s}}(0, \mu)$. If Hypothesis 5 holds, then $v \in W^{\mathrm{s}}(0, \mu) \cap \Sigma_{\text {out }}$ if and only if $\kappa v \in W^{\mathrm{s}}(0, \mu) \cap$ $\Sigma_{\text {out }}$; in particular, $g(\varphi, \mu)$ and $z(\varphi)$ are then $\pi$-periodic in $\varphi$ for all $(\varphi, \mu) \in U_{\Gamma}$.

Proof. Reversibility of (2.1) implies that $v \in W^{\mathrm{u}}(0, \mu)$ if and only if $\mathcal{R} v \in$ $W^{\mathrm{s}}(0, \mu)$. The first statement of the lemma now follows from Lemma 2.1 and the representation (2.5) of the reverser $\mathcal{R}$. Next, assume that (2.1) is equivariant under $\kappa$, which implies that $v=\left(v^{\mathrm{c}}, v^{\mathrm{s}}, \delta\right) \in W^{\mathrm{s}}(0, \mu) \cap \Sigma_{\text {out }}$ if and only if $\kappa v=\left(v^{\mathrm{c}}+\pi, v^{\mathrm{s}}, \delta\right) \in$ $W^{\mathrm{s}}(0, \mu) \cap \Sigma_{\text {out }}$. Hypothesis 6 shows that $v=\left(\varphi, v^{\mathrm{s}}, \delta\right) \in W^{\mathrm{s}}(0, \mu)$ if and only if $v^{\mathrm{s}}=g(\varphi, \mu)$. Applying this statement to $\varphi=v^{\mathrm{c}}$ and $\varphi=v^{\mathrm{c}}+\pi$, we can conclude that $g\left(v^{\mathrm{c}}, \mu\right)=v^{\mathrm{s}}=g\left(v^{\mathrm{c}}+\pi, \mu\right)$ for all $v^{\mathrm{c}}$. A similar argument shows that $z(\varphi)$ is periodic with period $\pi$. $\square$

In particular, both $g$ and $z$ are $\pi$-periodic in $\varphi$ if Hypothesis 5 is met, which explains why we imposed the conditions in Hypothesis 7 only modulo $\pi$ in this case. We record also that, on account of Hypothesis 6 , we have

$$
g(\varphi, z(\varphi)) \equiv 0 \quad \forall \varphi, \quad g(\varphi, \mu)=0 \quad \text { if and only if } \quad \mu=z(\varphi) .
$$

Using Lemmas 3.1 and 3.2, we can now construct symmetric and asymmetric homoclinic orbits to $u=0$ that pass near the periodic orbit $\gamma(\cdot, \mu)$.

4. Symmetric 1-pulses. In this section, we are interested in finding reversible homoclinic orbits $v(x)$ to $u=0$ that spend a long time near the periodic orbit $\gamma(\cdot, \mu)$. We focus on reversible 1-pulses which satisfy by definition

$$
\begin{aligned}
& v(x) \in \mathcal{V} \quad \text { for } \quad x \in[-L, L], \\
& v(L) \in W^{\mathrm{s}}(0, \mu) \cap \Sigma_{\text {out }}, \\
& v(0) \in \operatorname{Fix}(\mathcal{R}) \text { or } v(0) \in \operatorname{Fix}(\kappa \mathcal{R})
\end{aligned}
$$

for sufficiently large $L \gg 1$. Lemma 3.1 implies that (4.1) is met if and only if

$$
v(L)=\left(v^{\mathrm{c}}\left(L, \varphi_{0}, \mu\right), v^{\mathrm{s}}\left(L, \varphi_{0}, \mu\right), \delta\right) \in W^{\mathrm{s}}(0, \mu),
$$

where $\varphi_{0} \in \Theta$ is fixed. For $L \gg 1$, Hypothesis 6 shows that this equation is met if and only if

$$
\left(v^{\mathrm{c}}\left(L, \varphi_{0}, \mu\right), v^{\mathrm{s}}\left(L, \varphi_{0}, \mu\right)\right)=(\varphi, g(\varphi, \mu))
$$


for some $(\varphi, \mu) \in U_{\Gamma}$ or, equivalently,

$$
g\left(v^{\mathrm{c}}\left(L, \varphi_{0}, \mu\right), \mu\right)=v^{\mathrm{s}}\left(L, \varphi_{0}, \mu\right) .
$$

We have

$$
v^{\mathrm{c}}\left(L, \varphi_{0}, \mu\right) \stackrel{(3.2)}{=} \varphi_{0}+L+\mathrm{O}\left(\mathrm{e}^{-\eta L}\right), \quad \varphi_{0} \in \Theta \text { fixed }
$$

and define

$$
\ell:=v^{\mathrm{c}}\left(L, \varphi_{0}, \mu\right)-\varphi_{0}=L+\mathrm{O}\left(\mathrm{e}^{-\eta L}\right),
$$

where we treat $\ell$ as an element of the universal cover $\mathbb{R}$ of $S^{1}$. Thus, $\ell$ measures the phase in $\Sigma_{\text {out }}$, while $2 L$ is the time spent by the solution $v(x)$ near the periodic orbit $\gamma(\cdot, \mu)$. We can solve (4.3) uniquely for $L \gg 1$ to get

$$
L=L_{*}(\ell, \mu)=\ell+\mathrm{O}\left(\mathrm{e}^{-\eta \ell}\right), \quad \ell \gg 1, \quad \mu \in J .
$$

Substituting (4.4) into (4.2), we obtain the equation

$$
g\left(\varphi_{0}+\ell, \mu\right)=v^{\mathrm{s}}\left(L_{*}(\ell, \mu), \varphi_{0}, \mu\right) \stackrel{(3.2)}{=} \delta \mathrm{e}^{-2 \alpha(\mu) \ell}\left(1+\mathrm{O}\left(\mathrm{e}^{-\eta \ell}\right)\right) .
$$

It suffices now to solve (4.5) for $\ell \gg 1$ and $\mu \in J$, where $\varphi_{0} \in \Theta$ is fixed.

Lemma 4.1. Fix $\varphi_{0} \in \Theta$, then there exists an $\ell_{0} \gg 1$ so that (4.5) has a unique solution $\mu=\mu_{*}\left(\ell, \varphi_{0}\right) \in J$ for each $\ell>\ell_{0}$. Furthermore,

$$
\mu_{*}\left(\ell, \varphi_{0}\right)=z\left(\varphi_{0}+\ell\right)+\frac{\delta \mathrm{e}^{-2 \alpha\left(z\left(\varphi_{0}+\ell\right)\right) \ell}}{g_{\mu}\left(\varphi_{0}+\ell, z\left(\varphi_{0}+\ell\right)\right)}\left(1+\mathrm{O}\left(\mathrm{e}^{-\eta \ell}\right)\right)
$$

for all $\ell>\ell_{0}$. Near each $\ell_{*}$ with $z^{\prime}\left(\varphi_{0}+\ell_{*}\right)=0$, there is a unique fold bifurcation of the symmetric 1-pulses: the fold is nondegenerate and occurs at

$$
\ell=\ell_{*}+\left(\frac{2 \alpha}{g_{\mu} z^{\prime \prime}}+\frac{g_{\varphi \mu}}{g_{\mu}^{2} z^{\prime \prime}}\right) \delta \mathrm{e}^{-2 \alpha \ell_{*}}\left(1+\mathrm{O}\left(\mathrm{e}^{-\eta \ell_{*}}\right)\right),
$$

where all terms are evaluated at $\left(\varphi_{0}+\ell_{*}, z\left(\varphi_{0}+\ell_{*}\right)\right)$. No other fold bifurcations occur.

Proof. We set $\mu:=z\left(\varphi_{0}+\ell\right)+\tilde{\mu}$ so that (4.5) becomes

$$
g\left(\varphi_{0}+\ell, z\left(\varphi_{0}+\ell\right)+\tilde{\mu}\right)=\delta \mathrm{e}^{-2 \alpha\left(z\left(\varphi_{0}+\ell\right)+\tilde{\mu}\right) \ell}\left(1+\mathrm{O}\left(\mathrm{e}^{-\eta \ell}\right)\right) .
$$

On account of $(3.5), g(\varphi, \mu)=0$ if and only if $\mu=z(\varphi)$. Thus, the above equation can be written as

$$
\tilde{\mu}+\mathrm{O}\left(\tilde{\mu}^{2}\right)=\frac{\delta}{g_{\mu}\left(\varphi_{0}+\ell, z\left(\varphi_{0}+\ell\right)\right)} \mathrm{e}^{-2 \alpha\left(z\left(\varphi_{0}+\ell\right)+\tilde{\mu}\right) \ell}\left(1+\mathrm{O}\left(\mathrm{e}^{-\eta \ell}\right)\right),
$$

where we used that $\left|g_{\mu}\right| \geq b>0$ in $\Gamma$ by Hypothesis 6 . Using the implicit function theorem, we readily obtain (4.6). Fold bifurcations occur precisely when $\partial_{\ell} \mu_{*}\left(\ell, \varphi_{0}\right)=$ 0 . Inspecting (4.6), we find that folds can only occur near values $\ell_{*}$ for which $z^{\prime}\left(\varphi_{0}+\right.$ $\left.\ell_{*}\right)=0$, and a straightforward calculation gives (4.7) as the unique location of the fold near such an $\ell_{*}$. The fold is nondegenerate since $\partial_{\ell}^{2} \mu_{*}=z^{\prime \prime}\left(\varphi_{0}+\ell_{*}\right)+\mathrm{O}\left(\mathrm{e}^{-\eta \ell_{*}}\right) \neq 0$ by Hypothesis 7 .

Theorem 2.2 is a direct consequence of Lemma 4.1. 
5. Asymmetric 1-pulses. We now consider 1-pulses that are not necessarily symmetric. Throughout this section, we assume that Hypotheses 6-7 are met. We first consider the case where we do not have an additional $\mathbb{Z}_{2}$-symmetry, that is, we do not assume Hypothesis 5, and shall comment in $\$ 5.4$ below on the case where Hypothesis 5 is met.

Arbitrary 1-pulses correspond to solutions $v(x)$ with

$$
\begin{aligned}
& v(x) \in \mathcal{V} \quad \text { for } \quad x \in[-L, L], \\
& v(-L) \in W^{\mathrm{u}}(0, \mu) \cap \Sigma_{\text {in }}, \\
& v(L) \in W^{\mathrm{s}}(0, \mu) \cap \Sigma_{\text {out }}
\end{aligned}
$$

for sufficiently large $L \gg 1$. Using Lemmas 3.1 and 3.2, we see that (5.1) is equivalent to $\mathcal{G}(L, \varphi, \mu)=0$, where $\mathcal{G}$ is defined by

$$
\begin{aligned}
\mathcal{G}(L, \varphi, \mu) & :=\left(\begin{array}{c}
g\left(v^{\mathrm{c}}(L, \varphi, \mu), \mu\right)-v^{\mathrm{s}}(L, \varphi, \mu) \\
g\left(-v^{\mathrm{c}}(-L, \varphi, \mu), \mu\right)-v^{\mathrm{u}}(-L, \varphi, \mu)
\end{array}\right) \\
& \stackrel{(3.3)}{=}\left(\begin{array}{c}
g\left(v^{\mathrm{c}}(L, \varphi, \mu), \mu\right)-v^{\mathrm{s}}(L, \varphi, \mu) \\
g\left(v^{\mathrm{c}}(L,-\varphi, \mu), \mu\right)-v^{\mathrm{s}}(L,-\varphi, \mu)
\end{array}\right)
\end{aligned}
$$

and $\varphi \in S^{1}$ is arbitrary.

5.1. Pitchfork bifurcations from symmetric 1-pulses. We begin by investigating bifurcations from the symmetric 1-pulses. We set

$$
\begin{aligned}
\tilde{\mathcal{G}}(L, \varphi, \mu):=\left(\begin{array}{l}
\mathcal{G}_{1}(L, \varphi, \mu)+\mathcal{G}_{2}(L, \varphi, \mu) \\
\mathcal{G}_{1}(L, \varphi, \mu)-\mathcal{G}_{2}(L, \varphi, \mu)
\end{array}\right) \\
\stackrel{(5.2)}{=}\left(\begin{array}{c}
\mathcal{G}_{1}(L, \varphi, \mu)+\mathcal{G}_{1}(L,-\varphi, \mu) \\
\mathcal{G}_{1}(L, \varphi, \mu)-\mathcal{G}_{1}(L,-\varphi, \mu)
\end{array}\right),
\end{aligned}
$$

where $\left(\mathcal{G}_{1}, \mathcal{G}_{2}\right)$ are the components of $\mathcal{G}$ defined in $(5.2)$. Note that $\tilde{\mathcal{G}}(L,-\varphi, \mu)=$ $\rho \tilde{\mathcal{G}}(L, \varphi, \mu)$ for all $(L, \varphi)$, where $\rho$ is the reflection

$$
\rho=\left(\begin{array}{rr}
1 & 0 \\
0 & -1
\end{array}\right)
$$

In particular, $\tilde{\mathcal{G}}_{2}\left(L, \varphi_{0}, \mu\right) \equiv 0$ for the fixed points $\varphi_{0} \in \Theta=\{0, \pi\}$ of the $\mathbb{Z}_{2}$-action $\varphi \mapsto-\varphi$ on $S^{1}=[0,2 \pi] / \sim$, and the symmetric 1-pulses found in $\S 4$ correspond to solutions of $\tilde{\mathcal{G}}_{1}\left(L, \varphi_{0}, \mu\right)=0$ with $\varphi_{0} \in \Theta$. Bifurcations from symmetric 1-pulses occur only when

$$
\tilde{\mathcal{G}}_{1}\left(L, \varphi_{0}, \mu\right)=0, \quad \partial_{\varphi} \tilde{\mathcal{G}}_{2}\left(L, \varphi_{0}, \mu\right)=0
$$

for some $\varphi_{0} \in \Theta$, and the presence of the $\mathbb{Z}_{2}$-action given by $\varphi \mapsto-\varphi$ and $\rho$ shows that any such bifurcation will be a pitchfork bifurcation in the sense that solution branches will be invariant under the action $\varphi \mapsto-\varphi$. Inspecting (5.3), we see that (5.4) is equivalent to

$$
\mathcal{G}_{1}\left(L, \varphi_{0}, \mu\right)=0, \quad \partial_{\varphi} \mathcal{G}_{1}\left(L, \varphi_{0}, \mu\right)=0,
$$

that is, to

$$
\begin{aligned}
g\left(v^{\mathrm{c}}\left(L, \varphi_{0}, \mu\right), \mu\right)-v^{\mathrm{s}}\left(L, \varphi_{0}, \mu\right) & =0, \\
\left.\partial_{\varphi}\left[g\left(v^{\mathrm{c}}(L, \varphi, \mu), \mu\right)-v^{\mathrm{s}}(L, \varphi, \mu)\right]\right|_{\varphi=\varphi_{0}} & =0
\end{aligned}
$$


for some $\varphi_{0} \in \Theta$. The following lemma gives the location of all pitchfork bifurcations from symmetric 1-pulses and asserts that these lead to locally unique branches of asymmetric 1-pulses which are parametrized by $\varphi$ near $\varphi_{0}$.

Lemma 5.1. Fix $\varphi_{0} \in \Theta$, then there are numbers $\sigma>0$ and $\ell_{0} \gg 1$ with the following property. Near each $\ell_{*}>\ell_{0}$ with $z^{\prime}\left(\varphi_{0}+\ell_{*}\right)=0$, there is a unique pitchfork bifurcation of the symmetric 1-pulses, occurring for $\mu=\mu_{*}\left(\ell, \varphi_{0}\right)$ as in (4.6) and

$$
\ell=\ell_{*}+\frac{g_{\varphi \mu}}{g_{\mu}^{2} z^{\prime \prime}} \delta \mathrm{e}^{-2 \alpha \ell_{*}}\left(1+\mathrm{O}\left(\mathrm{e}^{-\eta \ell_{*}}\right)\right)
$$

where all terms are evaluated at $\left(\varphi_{0}+\ell_{*}, z\left(\varphi_{0}+\ell_{*}\right)\right)$ and $\ell$ is related to $L$ via (4.3). At these pitchfork bifurcations, a locally unique branch of asymmetric 1-pulses bifurcates, and this branch is parametrized smoothly by $\varphi$ for $\varphi \in U_{\sigma}\left(\varphi_{0}\right)$. No other bifurcations occur.

Proof. Equation (5.5) gives necessary conditions for bifurcations from symmetric 1-pulses. We have already solved the first equation of (5.5) in Lemma 4.1, obtaining

$$
\begin{aligned}
\mu=\mu_{*}\left(\ell, \varphi_{0}\right) & =z\left(\varphi_{0}+\ell\right)+\frac{\delta \mathrm{e}^{-2 \alpha\left(z\left(\varphi_{0}+\ell\right)\right) \ell}}{g_{\mu}\left(\varphi_{0}+\ell, z\left(\varphi_{0}+\ell\right)\right)}\left(1+\mathrm{O}\left(\mathrm{e}^{-\eta \ell}\right)\right) \\
& =: z\left(\varphi_{0}+\ell\right)+\tilde{\mu}(\ell)
\end{aligned}
$$

with $\ell$ given in (4.3). The remaining second equation of (5.5), given by

$$
\partial_{\varphi} \mathcal{G}_{1}\left(L, \varphi_{0}, \mu\right)=g_{\varphi}\left(v^{\mathrm{c}}\left(L, \varphi_{0}, \mu\right), \mu\right) v_{\varphi}^{\mathrm{c}}\left(L, \varphi_{0}, \mu\right)-v_{\varphi}^{\mathrm{s}}\left(L, \varphi_{0}, \mu\right)=0,
$$

is evaluated at $(L, \mu)=\left(L_{*}\left(\ell, \mu_{*}\left(\ell, \varphi_{0}\right)\right), \mu_{*}\left(\ell, \varphi_{0}\right)\right)$, and equations (3.2), (4.3), and (5.7) show that it is given by

$$
g_{\varphi}\left(\varphi_{0}+\ell, z\left(\varphi_{0}+\ell\right)+\tilde{\mu}(\ell)\right)\left(1+\mathrm{O}\left(\mathrm{e}^{-\eta \ell}\right)\right)-\mathrm{O}\left(\mathrm{e}^{-\left(2 \alpha\left(z\left(\varphi_{0}+\ell\right)\right)+\eta\right) \ell}\right)=0 .
$$

We expand as follows:

$$
\begin{aligned}
& g_{\varphi}\left(\varphi_{0}+\ell, z\left(\varphi_{0}+\ell\right)+\tilde{\mu}(\ell)\right) \\
& \quad=g_{\varphi}\left(\varphi_{0}+\ell, z\left(\varphi_{0}+\ell\right)\right)+g_{\varphi \mu}\left(\varphi_{0}+\ell, z\left(\varphi_{0}+\ell\right)\right) \tilde{\mu}(\ell)+\mathrm{O}\left(|\tilde{\mu}(\ell)|^{2}\right) \\
& \stackrel{(3.5)}{=}-g_{\mu}\left(\varphi_{0}+\ell, z\left(\varphi_{0}+\ell\right)\right) z^{\prime}\left(\varphi_{0}+\ell\right)+g_{\varphi \mu}\left(\varphi_{0}+\ell, z\left(\varphi_{0}+\ell\right)\right) \tilde{\mu}(\ell)+\mathrm{O}\left(|\tilde{\mu}(\ell)|^{2}\right)
\end{aligned}
$$

so that (5.8) becomes

$$
\begin{aligned}
& -g_{\mu}\left(\varphi_{0}+\ell, z\left(\varphi_{0}+\ell\right)\right) z^{\prime}\left(\varphi_{0}+\ell\right) \\
& +g_{\varphi \mu}\left(\varphi_{0}+\ell, z\left(\varphi_{0}+\ell\right)\right) \tilde{\mu}(\ell)+\mathrm{O}\left(\mathrm{e}^{-\left(2 \alpha\left(z\left(\varphi_{0}+\ell\right)\right)+\eta\right) \ell}\right)=0 .
\end{aligned}
$$

Since $g_{\mu}$ is bounded away from zero by Hypothesis 6 and $\tilde{\mu}(\ell) \rightarrow 0$ as $\ell \rightarrow \infty$, the above equation can be satisfied only near points $\ell_{*}$ with $z^{\prime}\left(\varphi_{0}+\ell_{*}\right)=0$. Setting $\ell=\ell_{*}+\tilde{\ell}$, expanding (5.9) around $\ell_{*}$, and using that $z^{\prime \prime}\left(\varphi_{0}+\ell_{*}\right) \neq 0$ by Hypothesis 7 gives (5.6).

To prove that a locally unique branch bifurcates at each pitchfork bifurcation and that this branch can be parametrized by $\varphi$ for $\varphi$ near $\varphi_{0}$, it suffices to prove that

$$
\frac{\mathrm{d}}{\mathrm{d} L}\left[\partial_{\varphi} \mathcal{G}_{1}\left(L, \varphi_{0}, \mu_{*}\left(L, \varphi_{0}\right)\right)\right] \neq 0 .
$$


Using (4.4) and (5.9), we find

$$
\begin{aligned}
& \frac{\mathrm{d}}{\mathrm{d} L}\left[\partial_{\varphi} \mathcal{G}_{1}\left(L, \varphi_{0}, \mu_{*}\left(L, \varphi_{0}\right)\right)\right] \\
& =-g_{\mu}\left(\varphi_{0}+\ell_{*}, z\left(\varphi_{0}+\ell_{*}\right)\right) z^{\prime \prime}\left(\varphi_{0}+\ell_{*}\right)+\mathrm{O}\left(\mathrm{e}^{-\eta \ell_{*}}\right)
\end{aligned}
$$

at the solution $\ell$ given in (5.6), and this expression is indeed bounded away from zero uniformly in $\ell_{*}$ by Hypotheses 6 and 7 . Finally, the neighbourhood $U_{\sigma}\left(\varphi_{0}\right)$ over which we can parametrize the branches in $\varphi$ is uniform in $\ell_{*}$, since the estimates in (5.9) and (5.10) and the dependence of (5.2) on $\varphi$ are uniform in $\ell_{*}$. $\square$

REMARK 1. We briefly compare the locations of saddle-node and pitchfork bifurcations given in Lemmas 4.1 and 5.1, respectively. Near each $\ell_{*}$ with $z^{\prime}\left(\varphi_{0}+\ell_{*}\right)=0$, there is a unique saddle-node at

$$
\ell_{\mathrm{sn}}=\ell_{*}+\left(\frac{2 \alpha}{g_{\mu} z^{\prime \prime}}+\frac{g_{\varphi \mu}}{g_{\mu}^{2} z^{\prime \prime}}\right) \delta \mathrm{e}^{-2 \alpha \ell_{*}}\left(1+\mathrm{O}\left(\mathrm{e}^{-\eta \ell_{*}}\right)\right)
$$

and a unique pitchfork at

$$
\ell_{\mathrm{pf}}=\ell_{*}+\frac{g_{\varphi \mu}}{g_{\mu}^{2} z^{\prime \prime}} \delta \mathrm{e}^{-2 \alpha \ell_{*}}\left(1+\mathrm{O}\left(\mathrm{e}^{-\eta \ell_{*}}\right)\right)
$$

where all terms are evaluated at $\left(\varphi_{0}+\ell_{*}, z\left(\varphi_{0}+\ell_{*}\right)\right)$, see (4.7) and (5.6), respectively. Thus, we have

$$
\ell_{\mathrm{sn}}=\ell_{\mathrm{pf}}+\frac{2 \alpha}{g_{\mu} z^{\prime \prime}} \delta \mathrm{e}^{-2 \alpha \ell_{*}}\left(1+\mathrm{O}\left(\mathrm{e}^{-\eta \ell_{*}}\right)\right),
$$

and the sign of the offset between these bifurcations is determined by the sign of the product $g_{\mu} z^{\prime \prime}$. The resulting scenarios are summarized in Figure 2.1.

5.2. Continuation of asymmetric 1-pulses. We showed in $\S 5.1$ that a locally unique branch of asymmetric 1-pulses bifurcates at each of the infinitely many pitchfork bifurcations identified in Lemma 5.1. In this section, we prove that each of these branches terminates at another pitchfork bifurcation, and we determine at which one.

To find asymmetric 1-pulses, we need to solve $\mathcal{G}(L, \varphi, \mu)=0$, where $\mathcal{G}$ is defined in (5.2). Lemma 3.1 implies that this equation is given by

$$
\begin{aligned}
& g\left(L+\varphi+\mathrm{O}\left(\mathrm{e}^{-\eta L}\right), \mu\right)=\mathrm{O}\left(\mathrm{e}^{-\eta L}\right), \\
& g\left(L-\varphi+\mathrm{O}\left(\mathrm{e}^{-\eta L}\right), \mu\right)=\mathrm{O}\left(\mathrm{e}^{-\eta L}\right),
\end{aligned}
$$

where $\varphi \in S^{1}$ is arbitrary, possibly after adjusting $\eta$ to a smaller positive value.

For $\varphi=\varphi_{0} \in \Theta$, we recover the symmetric 1-pulses. For each $\varphi_{0} \in \Theta$ and near each $L_{*}$ with $z^{\prime}\left(L_{*}+\varphi_{0}\right)=0$, Lemma 5.1 guarantees the existence of a locally unique branch of asymmetric solutions of (5.11) that are parametrized by $\varphi \in U_{\sigma}\left(\varphi_{0}\right)$. Furthermore, no other bifurcations to asymmetric 1-pulses occur. We are now interested in continuing the branches of asymmetric 1-pulses.

The first equation in (5.11) can be solved for $\mu$ as in $\S 4$, and we find

$$
\mu=z(L+\varphi)+\mathrm{O}\left(\mathrm{e}^{-\eta L}\right) .
$$

Using this expression, the second equation in (5.11) becomes

$$
g\left(L-\varphi+\mathrm{O}\left(\mathrm{e}^{-\eta L}\right), z(L+\varphi)+\mathrm{O}\left(\mathrm{e}^{-\eta L}\right)\right)=\mathrm{O}\left(\mathrm{e}^{-\eta L}\right) .
$$


We expect that the $\mathrm{O}\left(\mathrm{e}^{-\eta L}\right)$ terms in (5.12) will be of higher order. Omitting these terms for a moment, we arrive at the equation

$$
g(L-\varphi, z(L+\varphi))=0,
$$

which, on account of (3.5), has a solution $(L, \varphi)$ if and only if

$$
z(L-\varphi)=z(L+\varphi) \text {. }
$$

Recall that $z$ is $2 \pi$-periodic in its argument. Thus, for $\varphi \in \Theta,(5.14)$ is met for all $L$, and these solutions correspond to symmetric 1-pulses. In Lemma 5.1, we found all solutions $(L, \varphi)$ of $(5.11)$ near each $\left(L_{*}, \varphi_{0}\right)$ with $\varphi_{0} \in \Theta$ and $z^{\prime}\left(L_{*}+\varphi_{0}\right)=0$. Our goal is now to show that all other solutions of (5.11) can be found by solving (5.14).

LEMma 5.2. The function $\bar{g}(L, \varphi):=g(L-\varphi, z(L+\varphi))$ is $2 \pi$-periodic in both $\varphi$ and $L$, and we have $\nabla \bar{g}(L, \varphi) \neq 0$ at each point $(L, \varphi)$ for which $z(L-\varphi)=z(L+\varphi)$ except when both $\varphi \in \Theta$ and $z^{\prime}(L+\varphi)=0$.

Proof. We have

$$
\nabla \bar{g}(L, \varphi)=\left(\begin{array}{rr}
1 & 1 \\
-1 & 1
\end{array}\right)\left(\begin{array}{c}
g_{\varphi}(L-\varphi, z(L+\varphi)) \\
g_{\mu}(L-\varphi, z(L+\varphi)) z^{\prime}(L+\varphi)
\end{array}\right) .
$$

Equation (5.14) means that the argument $(L-\varphi, z(L+\varphi))=(L-\varphi, z(L-\varphi))$ lies in $\Gamma=\operatorname{graph}(z)$. Hypothesis 6 then implies that $\left|g_{\mu}(L-\varphi, z(L+\varphi))\right| \geq b>0$, and we can conclude that $\nabla \bar{g}(\varphi, L)=0$ if and only if both $z^{\prime}(L+\varphi)=0$ and $g_{\varphi}(L-\varphi, z(L+\varphi))=$ $g_{\varphi}(L-\varphi, z(L-\varphi))=0$. Using Hypothesis 6 and the definition of $g$, it is easy to see, via a Taylor expansion, that the second equation implies $z^{\prime}(L-\varphi)=0$. Thus, we have $z^{\prime}(L \pm \varphi)=0$ which, together with (5.14) and Hypothesis 7, implies $\varphi \in \Theta$ as claimed.

Lemma 5.2 will allow us below to solve (5.12) by the implicit function theorem near any solution $(L, \varphi)$ of (5.14) uniformly in $L \gg 1$, unless we have both $\varphi \in \Theta$ and $z^{\prime}(L+\varphi)=0$ in which case we already know all solutions by Lemma 5.1.

Thus, we initially focus on solving (5.14)

$$
Z(L, \varphi):=z(L+\varphi)-z(L-\varphi)=0
$$

for $(L, \varphi)$. We shall see that the shape of the graph of $z$ determines the entire solution structure of (5.15) and refer to Figure 1.7 for an illustration. We set $Q:=S^{1} \times[0, \pi]$ and

$$
\Lambda:=\{(L, \varphi) \in Q: Z(L, \varphi)=0\}, \quad \Lambda_{\text {bif }}:=\left\{(L, \varphi) \in \partial Q: z^{\prime}(L+\varphi)=0\right\} .
$$

Equation (5.15) readily shows that $\Lambda_{\text {bif }} \subset \partial Q \subset \Lambda$. Furthermore, choose $L_{M}$ and $L_{m}$ in $S^{1}$ so that

$$
M:=\max _{L \in S^{1}} z(L)=z\left(L_{M}\right), \quad m:=\min _{L \in S^{1}} z(L)=z\left(L_{m}\right)
$$

and note that $L_{M}$ and $L_{m}$ are unique in $S^{1}$ by Hypothesis 7. The following lemma contains the main result of this section, see also Figure 5.1.

Lemma 5.3. Assume that Hypotheses 6 and 7 are met, and let $k$ be the number of maxima of the function $z$.

(i) $\Lambda$ is the disjoint union of the one-dimensional manifolds $\partial Q=S^{1} \times\{0, \pi\}, \Lambda_{\mathrm{pf}}$ and $\Lambda_{\mathrm{iso}}: \Lambda_{\mathrm{pf}}$ is the disjoint union of the $2 k$ distinct global solution branches described in (ii) and (iii) below, and $\Lambda_{\text {iso }}$ consists of finitely many isolas, each diffeomorphic to a circle, whose projection onto the L-axis is not onto. 

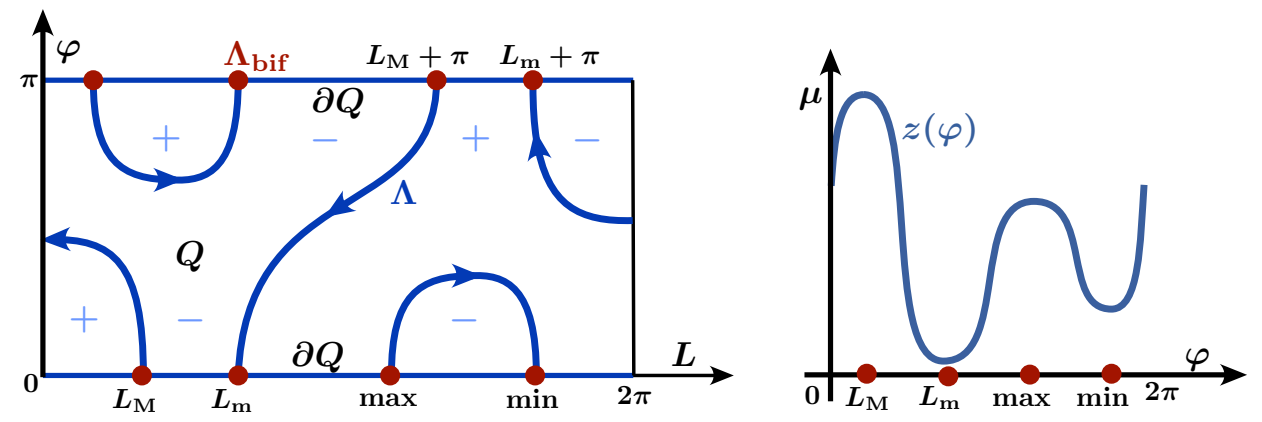

Fig. 5.1. The set $\Lambda=Z^{-1}(0)$ corresponding to the function $z$ displayed in the right panel is shown together with the sign of $Z$ in the components of $Q \backslash \Lambda$. The set $\Lambda_{\mathrm{bif}}$ is the union of the bullets located at $(L, 0)$ and $(L, \pi)$ which correspond to maxima and minima of $z(L+\varphi)$. In general, $\Lambda$ may also contain isolas.

(ii) Each element $\left(L_{*}, 0\right)$ and $\left(L_{*}, \pi\right)$ of $\Lambda_{\mathrm{bif}}$ is a generic pitchfork bifurcation point, giving rise to a unique global branch of solutions of (5.15) in $Q$. These branches do not cross, and they begin and end in $\Lambda_{\mathrm{bif}}$.

(iii) The branches starting at $\left(L_{m}, 0\right)$ and $\left(L_{M}, 0\right)$ terminate at $\left(L_{M}-\pi, \pi\right)$ and $\left(L_{m}-\pi, \pi\right)$, respectively. Each branch starting at $\left(L_{1}, 0\right)$, where $L_{1} \neq L_{M}$ is a non-global maximum of $z$, terminates at a point $\left(L_{2}, 0\right)$ where $L_{2} \neq L_{m}$ is a non-global minimum of $z$. Similarly, branches starting at $\left(L_{1}, \pi\right)$, where $L_{1} \neq L_{M}$ is a non-global maximum of $z$, terminate at points $\left(L_{2}, \pi\right)$, where $L_{2} \neq L_{m}$ is a non-global minimum of $z$.

Proof. We record that

$$
\nabla Z(L, \varphi)=\left(\begin{array}{rr}
1 & -1 \\
1 & 1
\end{array}\right)\left(\begin{array}{l}
z^{\prime}(L+\varphi) \\
z^{\prime}(L-\varphi)
\end{array}\right)
$$

and consider the planar Hamiltonian vector field

$$
\left(\begin{array}{l}
L_{s} \\
\varphi_{s}
\end{array}\right)=F(L, \varphi):=\left(\begin{array}{rr}
0 & 1 \\
-1 & 0
\end{array}\right) \nabla Z(L, \varphi)
$$

associated with the energy $Z(L, \varphi)$, whose zero level set is $\Lambda$. Note that $\nabla Z$ vanishes at $(L, \varphi) \in \Lambda$ if and only if both $z^{\prime}(L+\varphi)=0$ and $z^{\prime}(L-\varphi)=0$. Hypothesis 7 asserts that this can only happen when $\varphi=0$ or $\varphi=\pi$, that is, when $(L, \varphi) \in \Lambda_{\text {bif }}$. Thus, all equilibria of (5.16) in $\Lambda$ lie in $\Lambda_{\text {bif }}$, and they are hyperbolic saddles since

$$
\mathrm{D} F\left(L, \varphi_{0}\right)=2\left(\begin{array}{cc}
z^{\prime \prime}\left(L-\varphi_{0}\right) & 0 \\
0 & -z^{\prime \prime}\left(L-\varphi_{0}\right)
\end{array}\right), \quad \varphi_{0} \in\{0, \pi\}
$$

at each such equilibrium. Since $\nabla Z$ is non-zero everywhere else on $\Lambda$, it follows from Poincaré-Bendixson that $\Lambda \backslash \Lambda_{\text {bif }}$ is a one-dimensional manifold, namely the union of the heteroclinic orbits that emanate from and terminate at $\Lambda_{\text {bif }}$ and finitely many periodic orbits. This proves (ii). We have also shown all claims made in (i) except that we have not yet proved that isolas cannot extend from $L=0$ to $L=2 \pi$. This will follow at once from (iii) since the branches that connect global extrema serve as a barrier; see Figure 5.1.

If $\left(L, \varphi_{0}\right) \in \Lambda_{\text {bif }}$ corresponds to a maximum of $z$, then the eigenvalue belonging to the eigenvector $(0,1)$ is positive, and it is the unstable manifold of the equilibrium that 

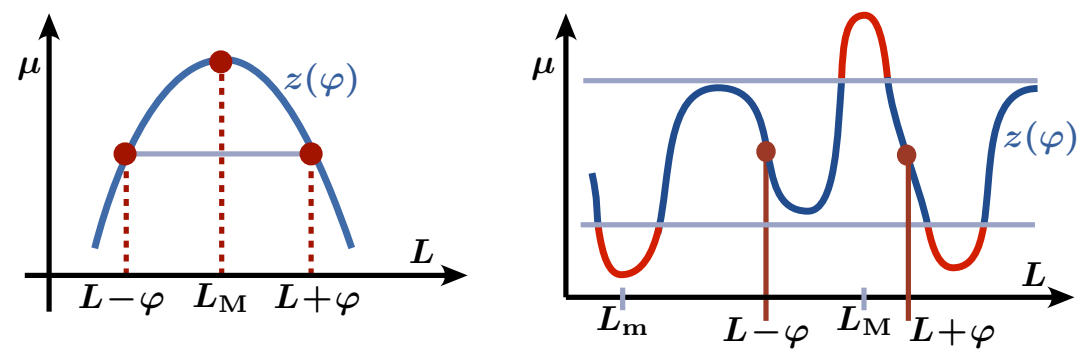

FIG. 5.2. The left panel shows representative solutions $L \pm \varphi$ of $z(L+\varphi)=z(L-\varphi)$ near the pitchfork bifurcation from $L=L_{\mathrm{M}}$. The right panel illustrates that the solutions $L-\varphi$ and $L+\varphi$ are trapped in the intervals $\left(L_{\mathrm{m}}, L_{\mathrm{M}}\right)$ and $\left(L_{\mathrm{M}}, L_{\mathrm{m}}+2 \pi\right)$, respectively, with $0<\varphi<\pi$ until either $L-\varphi=L_{\mathrm{m}}$ and $L+\varphi=L_{\mathrm{m}}+2 \pi$ (which implies $\varphi=\pi$ ), or else $L=L_{\mathrm{M}}$ and $\varphi=0$.

evolves into the interior ${ }_{Q}$ of $Q$. Similarly, at minima of $z$, it is the stable manifold that points into $\stackrel{Q}{Q}$. Thus, orbits emanating from maxima of $z$ must terminate at minima of $z$, and vice versa; see also Figure 5.1. This proves part of (iii).

Consider now the pitchfork branch emerging from $(L, \varphi)=\left(L_{M}, 0\right)$ where $z\left(L_{M}\right)$ is the uniquely assumed maximal value of $z$. We wish to prove that this branch terminates at $(L, \varphi)=\left(L_{m} \pm \pi, \pi\right)$. To prove this assertion, note that $L-\varphi<L_{M}<$ $L+\varphi$ near the bifurcation point, see Figure 5.2. Thus, inspecting Figure 5.2, we see that there are precisely two possibilities: first, $L-\varphi$ or $L+\varphi$ reach $L_{m}$ or $L_{m}+2 \pi$ at some point, in which case both must assume these values as $L_{m}$ is the only value modulo $2 \pi$ for which $z(L)=z\left(L_{m}\right)$; in this case, $\varphi=\pi$, and the branch terminates at $L=L_{m} \pm \pi$. The second option is that $L-\varphi$ or $L+\varphi$ reach $L_{M}$, in which case they both must assume this value together; this is ruled out though by the vector-field interpretation discussed above. Thus, only the first possibility can occur which proves the assertion.

Next, we consider a branch emerging from $(L, \varphi)=\left(L_{*}, 0\right)$ where $L_{*}$ corresponds to a non-global maximum or minimum of $z$. Without loss of generality, we may assume that $L_{*} \in\left(L_{m}, L_{M}\right)$. We conclude that $L-\varphi$ and $L+\varphi$ can never leave the interval $\left(L_{m}, L_{M}\right)$ along the branch for $\varphi>0$ since $m<z(L-\varphi)=z(L+\varphi)<M$ for all $L \pm \varphi \in\left(L_{m}, L_{M}\right)$, see also Figure 5.2. Thus, the branch must terminate at $\varphi=0$ as claimed. This finishes the proof of (iii). $\square$

The results established in Lemma 5.3 are valid for (5.14) and therefore also for (5.13). We need to lift these results to the full problem (5.12) given by

$$
g\left(L-\varphi+\mathrm{O}\left(\mathrm{e}^{-\eta L}\right), z(L+\varphi)+\mathrm{O}\left(\mathrm{e}^{-\eta L}\right)\right)=\mathrm{O}\left(\mathrm{e}^{-\eta L}\right)
$$

By decomposing $L=\ell+2 \pi n$ uniquely for $\ell \in S^{1}$ and $n \in \mathbb{N}$, we arrive at the equivalent system

$$
g\left(\ell-\varphi+\mathrm{O}\left(\mathrm{e}^{-\eta n}\right), z(\ell+\varphi)+\mathrm{O}\left(\mathrm{e}^{-\eta n}\right)\right)=\mathrm{O}\left(\mathrm{e}^{-\eta n}\right), \quad(\varphi, \ell) \in Q .
$$

Lemma 5.1 shows that $\Lambda$ and the solution set of (5.17) are diffeomorphic in $U_{\sigma}\left(\Lambda_{\text {bif }}\right)$ for all $n$ sufficiently large. Outside this neighbourhood of $\Lambda_{\text {bif }}$, Lemmas 5.2 and 5.3 show that the solution set of (5.17) is diffeomorphic to $\Lambda$, since $\Lambda=Z^{-1}(0)$, zero is a regular value of $Z$ when restricted to the complement of $U_{\sigma}\left(\Lambda_{\text {bif }}\right)$, and no additional solutions can appear for (5.17) due to compactness of $Q$. This completes the proof of Theorem 2.3 . 
5.3. Isolas of asymmetric 1-pulses. We show that isolas of asymmetric 1pulses can occur for appropriate functions $z$.

Lemma 5.4. Assume that Hypotheses 6 and 7 are met, and let $k$ be the number of maxima of the function $z$. For $k=1,2$, the set $\Lambda=Z^{-1}(0)$ discussed in Lemma 5.3 is connected, and therefore does not contain any isolas. For $k \geq 3$, isolas of asymmetric 1-pulses may exist; an example of a function $z$ for which an isola exists is given in Figure 1.8.

Proof. Using the procedure outlined above in Figure 1.7, it is easy to check that isolas cannot exist for functions $z$ with one or two maxima. We omit a formal proof, which can be obtained using the arguments given in the proof of Lemma 5.3. Figure 1.8 gives an example of a function $z$ that admits an isola. Lemma 5.3 shows that the drawn isola is necessarily a manifold diffeomorphic to $S^{1}$.

5.4. $\mathbb{Z}_{2}$-symmetry. Finally, we comment on $\mathbb{Z}_{2}$-equivariant systems for which Hypothesis 5 is met. In this case, Lemma 3.2 implies that $g$, and therefore $z$, are periodic in $\varphi$ with period $\pi$. Thus, we can repeat the analysis carried out above for period $\pi$ instead of $2 \pi$ or, alternatively, simply factor out the $\mathbb{Z}_{2}$-action near the periodic orbits and apply our results to the system on the $\mathbb{Z}_{2}$-orbit space. Either way, we find that the branches emerging from global maxima and minima connect $\varphi=0$ to $\varphi= \pm \pi / 2$ and $\varphi=\pi / 2$ to $\varphi=0$ and $\varphi=\pi$.

6. Extensions. We discuss and prove various extensions of our results. Isolas of fronts are considered in $\S 6.1$, while higher-dimensional systems and extensions to $2 \mathrm{D}$ patterns such as those shown in Figure 1.2 are treated in $\S 6.2$ and $\S 6.3$, respectively. Snaking in reversible systems without a conserved quantity is studied in $\S 6.4$.

6.1. Isolas of fronts. Hypothesis 6 excludes the case illustrated in Figure 1.5(iii), where the fronts between rolls and the trivial state lie on an isola in parameter space. The expected bifurcation diagram, shown in Figure 1.6(iii), predicts isolas of symmetric pulses, and such isolas have indeed been observed recently in [3, Figure 24] for the Swift-Hohenberg equation (1.1). We assumed in Hypothesis 6 that the set $\Gamma$, defined in $(2.6)$ as

$$
\Gamma:=\left\{(\varphi, \mu) \in S^{1} \times J: W^{\mathrm{s}}(0, \mu) \cap W^{\mathrm{uu}}(\gamma(\varphi, \mu), \mu) \cap \Sigma_{\mathrm{out}} \neq \emptyset\right\},
$$

is a graph $(\varphi, z(\varphi))$ and that we can parametrize $W^{\mathrm{s}}(0, \mu) \cap \Sigma_{\text {out }}$ over $(\varphi, \mu)$. A natural generalization is to assume that there is a smooth function $G: S^{1} \times I \times J \rightarrow \mathbb{R}$ so that

$$
\left(\varphi, v^{\mathrm{s}}\right) \in W^{\mathrm{s}}(0, \mu) \cap \Sigma_{\text {out }} \quad \Longleftrightarrow G\left(\varphi, v^{\mathrm{s}}, \mu\right)=0
$$

with $\Gamma=\left\{(\varphi, \mu) \in S^{1} \times J: G(\varphi, 0, \mu)=0\right\} \subset S^{1} \times \stackrel{\circ}{J}$, which precludes that $\Gamma$ intersects $S^{1} \times \partial J$. Note that if Hypothesis 6 is met, we have $G\left(\varphi, v^{\mathrm{s}}, \mu\right)=g(\varphi, \mu)-v^{\mathrm{s}}$. The nondegeneracy conditions

$$
\nabla_{(\varphi, \mu)} G(\varphi, 0, \mu) \neq 0, \quad \nabla_{\left(\varphi, v^{\mathrm{s}}\right)} G(\varphi, 0, \mu) \neq 0 \quad \forall(\varphi, \mu) \in \Gamma
$$

guarantee that $\Gamma$ is a manifold and that $W^{\mathrm{s}}(0, \mu) \cap \Sigma_{\text {out }}$ is, locally near each element of $\Gamma$, the graph of a smooth function that depends on $\left(v^{\mathrm{s}}, \mu\right)$ or on $(\varphi, \mu)$.

THEOREM 6.1. In the situation described above, assume that (6.1) and Hypotheses 1-4, and possibly Hypothesis 5, are met. For all points $(\varphi, \mu) \in \Gamma$ near which we can parametrize $\Gamma$ over $\varphi$, we also assume that Hypothesis 7 is met. There are then constants $\eta>0$ and $L_{*} \gg 1$ so that, for each $L>L_{*}$ and $\varphi_{0} \in \Theta$, (2.1) has a 
symmetric 1-pulse for $\mu \in J$ that spends time $2 L$ in the neighborhood $\mathcal{V}$ near $\gamma(\cdot, \mu)$ if and only if $(L, \mu) \in \mathcal{S}_{\varphi_{0}}$, where $\mathcal{S}_{\varphi_{0}}$ is a smooth one-dimensional manifold, which is $\mathrm{O}\left(\mathrm{e}^{-\eta L}\right)$ close in $\mathcal{C}^{1}$ to the manifold

$$
\left\{(\mu, L) \in J \times\left(L_{*}, \infty\right): G\left(L+\varphi_{0}, 0, \mu\right)=0\right\} .
$$

Furthermore, (2.1) has branches of asymmetric 1-pulses that can be constructed as illustrated in Figure 1.7 and discussed in $\S 5$. If $\Gamma$ is diffeomorphic to $S^{1}$ and the projection of $\Gamma$ onto its first component $\varphi \in S^{1}$ is not onto, then $\mathcal{S}_{\varphi_{0}}$ is the union of infinitely many disjoint circles, and branches of asymmetric states that emerge and terminate at maxima and minima of $\Gamma$ connect each of these circles to itself.

In particular, if $\Gamma$ is an isola, then isolas of symmetric pulses for different $\varphi_{0}$ are not connected, see Figure 1.6, in contrast to the situation discussed in Theorem 2.3.

Proof. The proofs given in $\S 4$ and $\S 5$ need only minor modifications which we now outline. For symmetric pulses, the bifurcation equation (4.2) is replaced by

$$
G\left(v^{\mathrm{c}}\left(L, \varphi_{0}, \mu\right), v^{\mathrm{s}}\left(L, \varphi_{0}, \mu\right), \mu\right)=G\left(\varphi_{0}+L+\mathrm{O}\left(\mathrm{e}^{-\eta L}\right), \mathrm{O}\left(\mathrm{e}^{-\eta L}\right), \mu\right)=0 .
$$

The first nondegeneracy condition in (6.1) allows us to use the implicit function theorem to solve the above equation uniquely near each $(L, \mu)$ with $(L \bmod 2 \pi, \mu) \in \Gamma$. Pitchfork bifurcations from symmetric to asymmetric states occur near elements $(\varphi, \mu) \in \Gamma$ with $\partial_{\varphi} G(\varphi, 0, \mu)=0$ : equation (6.1) ensures that $W^{\mathrm{s}}(0, \mu)$ can be parametrized via $v^{\mathrm{s}}=g(\varphi, \mu)$ near such points, and we are therefore back in the setting of $\S 5.1$. Branches of asymmetric states can also be continued: (5.11) is now given by

$$
G\left(L+\varphi+\mathrm{O}\left(\mathrm{e}^{-\eta L}\right), \mathrm{O}\left(\mathrm{e}^{-\eta L}\right), \mu\right)=0, \quad G\left(L-\varphi+\mathrm{O}\left(\mathrm{e}^{-\eta L}\right), \mathrm{O}\left(\mathrm{e}^{-\eta L}\right), \mu\right)=0
$$

and it is easy to verify that the Jacobian with respect to $(L, \varphi, \mu)$ of the left-hand side has full rank except at pitchfork bifurcation points. This shows that solutions of the asymptotic problem, obtained by omitting the $\mathrm{O}\left(\mathrm{e}^{-\eta L}\right)$ terms in (6.2), persist for $(6.2)$.

6.2. Higher-dimensional ODEs. We focus now on generalizations of the results in $\S 2$ to higher-dimensional systems where $u \in \mathbb{R}^{2 n}$ with $n>2$. In this situation, the parametrization of invariant manifolds is slightly different. We assume that the linearization of the time- $2 \pi$ map of the periodic orbits has two simple real Floquet multipliers at $\mathrm{e}^{ \pm 2 \pi \alpha(\mu)}$ with $\alpha(\mu)>0$ for all $\mu \in J$ and that there is an $\eta>0$ so that the remaining Floquet multipliers have modulus either less than $\mathrm{e}^{-2 \pi(\alpha(\mu)+\eta)}$ or larger than $\mathrm{e}^{2 \pi(\alpha(\mu)+\eta)}$ uniformly in $\mu \in J$. We can now choose coordinates $\left(v^{\mathrm{c}}, v^{\mathrm{s}}, v^{\mathrm{ss}}, v^{\mathrm{u}}, v^{\mathrm{uu}}\right)$ near the periodic orbits $\gamma(x, \mu)$ in the zero energy level set that reflect the uniform spectral decomposition assumed above. In particular, $W^{\mathrm{u}}(\gamma(x, \mu), \mu)$ is given by $\left(v^{\mathrm{c}}, 0,0, v^{\mathrm{u}}, v^{\mathrm{uu}}\right)$ in these coordinates, and we can define the section $\Sigma_{\text {out }}$ by requiring $v^{\mathrm{u}}=\delta$. The key hypothesis is that

$$
\begin{aligned}
W^{\mathrm{s}}(0, \mu) \cap \Sigma_{\text {out }}= & \left\{\left(v^{\mathrm{c}}, v^{\mathrm{s}}, v^{\mathrm{ss}}, \delta, v^{\mathrm{uu}}\right)=\left(v^{\mathrm{c}}, g^{\mathrm{s}}\left(v^{\mathrm{c}}, v^{\mathrm{ss}}, \mu\right), v^{\mathrm{ss}}, \delta, g^{\mathrm{uu}}\left(v^{\mathrm{c}}, v^{\mathrm{ss}}, \mu\right)\right)\right. \\
& \left.\left(v^{\mathrm{c}}, v^{\mathrm{ss}}, \mu\right) \in S^{1} \times B \times J\right\}
\end{aligned}
$$

for appropriate smooth functions $g^{\mathrm{s}}$ and $g^{\mathrm{uu}}$, where $B$ is a small ball centered at the origin in $\mathbb{R}^{n-1}$. In particular, zeros of $g^{\mathrm{s}}$ correspond to heteroclinic orbits between $u=0$ and $u=\gamma(x, \mu)$. Note that (6.3) requires that the stable manifold $W^{\mathrm{s}}(0, \mu)$ approaches the unstable manifold $W^{\mathrm{u}}(\gamma(x, \mu), \mu)$ of the periodic orbits in backward time 
along the direction associated with the unstable leading Floquet multiplier $\mathrm{e}^{2 \pi \alpha(\mu)}$ and that $W^{\mathrm{s}}(0, \mu)$ intersects $W^{\mathrm{u}}(\gamma(x, \mu), \mu)$ as transversely as possible.

The results in [40, Theorem 2.1 and (2.8)] show that there is an $\eta>0$ so that Lemma 3.1 remains true provided (3.2) is replaced by

$$
\begin{aligned}
v(-L) & =\left(\varphi-L+\mathrm{O}\left(\mathrm{e}^{-\eta L}\right), \delta, v^{\mathrm{ss}}+\mathrm{O}\left(\mathrm{e}^{-\eta L}\right), \mathrm{O}\left(\mathrm{e}^{-\eta L}\right), \mathrm{O}\left(\mathrm{e}^{-\eta L}\right)\right), \\
v(L) & =\left(\varphi+L+\mathrm{O}\left(\mathrm{e}^{-\eta L}\right), \mathrm{O}\left(\mathrm{e}^{-\eta L}\right), \mathrm{O}\left(\mathrm{e}^{-\eta L}\right), \delta, v^{\mathrm{uu}}+\mathrm{O}\left(\mathrm{e}^{-\eta L}\right)\right), \\
v(0) & =\left(\varphi, \mathrm{O}\left(\mathrm{e}^{-\eta L}\right), \mathrm{O}\left(\mathrm{e}^{-\eta L}\right), \mathrm{O}\left(\mathrm{e}^{-\eta L}\right), \mathrm{O}\left(\mathrm{e}^{-\eta L}\right)\right) .
\end{aligned}
$$

To find symmetric 1-pulses, we need to solve

$$
\begin{aligned}
& \left(v^{\mathrm{c}}, g^{\mathrm{s}}\left(v^{\mathrm{c}}, v^{\mathrm{ss}}, \mu\right), v^{\mathrm{ss}}, \delta, g^{\mathrm{uu}}\left(v^{\mathrm{c}}, v^{\mathrm{ss}}, \mu\right)\right) \\
& =\left(\varphi+L+\mathrm{O}\left(\mathrm{e}^{-\eta L}\right), \mathrm{O}\left(\mathrm{e}^{-\eta L}\right), \mathrm{O}\left(\mathrm{e}^{-\eta L}\right), \delta, v^{\mathrm{uu}}+\mathrm{O}\left(\mathrm{e}^{-\eta L}\right)\right)
\end{aligned}
$$

which, upon solving for $\left(v^{\mathrm{ss}}, v^{\mathrm{uu}}\right)$, reduces to

$$
\left(v^{\mathrm{c}}, g^{\mathrm{s}}\left(v^{\mathrm{c}}, v^{\mathrm{ss}}, \mu\right)\right)=\left(\varphi+L+\mathrm{O}\left(\mathrm{e}^{-\eta L}\right), \mathrm{O}\left(\mathrm{e}^{-\eta L}\right)\right) .
$$

A similar reduction works for asymmetric 1-pulses.

We conclude that the results presented in $\S 2$ remain true for systems in $\mathbb{R}^{2 n}$, provided we replace all terms of the form $\mathrm{e}^{-2 \alpha(\mu) \ell}$ by $\mathrm{O}\left(\mathrm{e}^{-\eta \ell}\right)$. Thus, while we lose information about the specific location of saddle-node and pitchfork bifurcations and about the specific offset of the snaking curves, the results about the bifurcation structure remain valid.

6.3. Snaking in the planar Swift-Hohenberg equation. We consider stationary planar patterns that are periodic and reflection-symmetric in one spatial direction and localized in the other direction, and refer to Figure 1.2 or [4, Figure 15a] for examples. As shown in Figure 1.2, snaking is possible for these patterns, and our goal is to show that the results described in $\S 2$ apply to such patterns under assumptions similar to those discussed in $\S 6.2$.

For the sake of clarity, we outline the framework within which we can study such patterns only for the planar Swift-Hohenberg equation

$$
U_{t}=-(1+\Delta)^{2} U-\mu U+\nu U^{2}-U^{3}
$$

with $(x, y) \in \mathbb{R}^{2}$. We assume that $U(x, y)$ is a smooth solution of $(6.4)$ that is periodic in $y$ with minimal period $\ell>0$. We define the wave number $\kappa=2 \pi / \ell$ and introduce the rescaled variable $\phi=\kappa y$, which allows us to consider the equation

$$
\partial_{x}^{4} U+2 \kappa^{2} \partial_{x}^{2} \partial_{\phi}^{2} U+\kappa^{4} \partial_{\phi}^{4} U+2\left(\partial_{x}^{2} U+\kappa^{2} \partial_{\phi}^{2} U\right)+(1+\mu) U-\nu U^{2}+U^{3}=0
$$

with $\phi \in S^{1}=[0,2 \pi] / \sim$. For fixed $\kappa$ and $\nu$, we write this equation as a first-order system in $x$ and obtain

$$
u_{x}=A(\mu) u+N(u)
$$

where

$$
\begin{aligned}
& A(\mu)=\left(\begin{array}{cccc}
0 & 1 & 0 & 0 \\
0 & 0 & 1 & 0 \\
0 & 0 & 0 & 1 \\
-\kappa^{4} \partial_{\phi}^{4}-2 \kappa^{2} \partial_{\phi}^{2}-(1+\mu) & 0 & -2-2 \kappa^{2} \partial_{\phi}^{2} & 0
\end{array}\right), \\
& N(u)=\left(\begin{array}{c}
0 \\
0 \\
0 \\
\nu U^{2}-U^{3}
\end{array}\right) \text {, }
\end{aligned}
$$


and

$$
u=\left(U, U_{x}, U_{x x}, U_{x x x}\right) \in Y:=H_{\mathrm{e}}^{3}\left(S^{1}\right) \times H_{\mathrm{e}}^{2}\left(S^{1}\right) \times H_{\mathrm{e}}^{1}\left(S^{1}\right) \times L_{\mathrm{e}}^{2}\left(S^{1}\right)
$$

with $L_{\mathrm{e}}^{2}\left(S^{1}\right)=\left\{u \in L^{2}\left(S^{1}\right): u(\phi)=u(-\phi)\right\}$. In other words, for each $x, u(x)$ is an even periodic function of $\phi$. We record that $A(\mu)$ is closed and densely defined on $Y$ with domain $Y^{1}=H_{\mathrm{e}}^{4} \times H_{\mathrm{e}}^{3} \times H_{\mathrm{e}}^{2} \times H_{\mathrm{e}}^{1}$, and for each $\mu>0$ there is a constant $C>0$ so that the resolvent estimate

$$
\left\|(A(\mu)-\mathrm{i} k)^{-1}\right\|_{L(Y)} \leq \frac{C}{1+|k|} \quad \forall k \in \mathbb{R}
$$

holds. Note also that the nonlinearity $N: Y \rightarrow Y$ is smooth due to Sobolev's embedding theorem. In the remainder of this section, we will denote by $|\cdot|$ the norm in $Y$.

Localized structures such as those shown in Figure 1.2 correspond to homoclinic orbits of (6.5) in $x$ that connect the trivial state $u=0$ to itself in the space $Y$ of periodic functions in $\phi$, and we are therefore in a familiar setting. As in $\S 6.2$, Lemma 3.1 is the key technical result that we need to carry forward our analysis, and much of this section will deal with extending Lemma 3.1 to (6.5).

We first discuss the analogues of Hypotheses 1-7 that we need to analyse symmetric and asymmetric patterns. As shown in [23, §3.3], (6.5) is reversible with reverser $\mathcal{R} u=\mathcal{R}\left(u_{1}, u_{2}, u_{3}, u_{4}\right)=\left(u_{1},-u_{2}, u_{3},-u_{4}\right)$ and conservative with first integral

$$
\begin{aligned}
\mathcal{H}(u, \mu)= & \int_{0}^{2 \pi}\left[u_{2} u_{4}-\frac{u_{3}^{2}}{2}+u_{2}^{2}+\frac{(1+\mu) u_{1}^{2}}{2}-\frac{\nu u_{1}^{3}}{3}+\frac{u_{1}^{4}}{4}\right. \\
& \left.-\kappa^{2}\left(\partial_{y} u_{2}\right)^{2}-\kappa^{2}\left(\partial_{y} u_{1}\right)^{2}+\frac{\kappa^{4}\left(\partial_{y}^{2} u_{1}\right)^{2}}{2}\right] \mathrm{d} y .
\end{aligned}
$$

We infer from (6.6) that the origin is hyperbolic for $\mu>0$ as required in Hypothesis 3.

We need to assume the existence of a family $\gamma(x, \mu)$ of symmetric periodic solutions of (6.5) that have zero energy so that $\mathcal{H}(\gamma(\cdot, \mu), \mu)=0$ for all $\mu$ in a closed nontrivial interval $J \subset \mathbb{R}^{+}$. Furthermore, we assume that the gradient of $\mathcal{H}$ does not vanish along the family $\gamma(0, \mu)$ and that the Floquet spectrum of the orbits $\gamma(x, \mu)$ is as described in $\S 6.2$, where we remark that [25] ensures that these spectra are well defined for solutions of (6.5). The results proved in $[2,26,23]$ show that this analogue of Hypothesis 4 is satisfied by both rolls and hexagons provided $0<\mu \ll 1$. If the cubic-quintic nonlinearity $U \mapsto \nu U^{3}-U^{5}$ is used in (6.4), Hypothesis 5 is also satisfied, at least for $0<\mu \ll 1$.

It remains to discuss Hypotheses 6-7 which are concerned with the invariant manifolds of the origin $u=0$ and the periodic orbits $\gamma(x, \mu)$. Even though (6.5) is ill-posed as an initial-value problem, the theory developed in [28, 39] shows that stable and unstable manifolds exist. Moreover, [39, Theorem 5.1] shows that the stable and unstable manifolds of $\gamma$ are foliated by smooth strong stable and strong unstable fibers. Since the zero-energy level set is a smooth manifold near the periodic orbits, we can flatten it. Using the smooth invariant foliations, we can then define coordinates $v=\left(v^{\mathrm{c}}, v^{\mathrm{s}}, v^{\mathrm{ss}}, v^{\mathrm{u}}, v^{\mathrm{uu}}\right)$ near the periodic orbits $\gamma(x, \mu)$ in the zero-energy level set as in $\S 6.2$. In these coordinates, we assume that there exist smooth functions $g^{j}$ for $j=\mathrm{u}, \mathrm{uu}, \mathrm{s}, \mathrm{ss}$ so that we have

$$
W^{\mathrm{s}}(0, \mu) \cap \Sigma_{\text {out }}=\left\{v=\left(v^{\mathrm{c}}, g^{\mathrm{s}}\left(v^{\mathrm{c}}, v^{\mathrm{ss}}, \mu\right), v^{\mathrm{ss}}, \delta, g^{\mathrm{uu}}\left(v^{\mathrm{c}}, v^{\mathrm{ss}}, \mu\right)\right):\right.
$$




$$
\begin{aligned}
& \left.\left(v^{\mathrm{c}}, v^{\mathrm{ss}}, \mu\right) \in S^{1} \times B \times J\right\}, \\
& W^{\mathrm{u}}(0, \mu) \cap \Sigma_{\mathrm{in}}=\left\{v=\left(v^{\mathrm{c}}, \delta, g^{\mathrm{ss}}\left(v^{\mathrm{c}}, v^{\mathrm{uu}}, \mu\right), g^{\mathrm{u}}\left(v^{\mathrm{c}}, v^{\mathrm{uu}}, \mu\right), v^{\mathrm{uu}}\right):\right. \\
& \left.\quad\left(v^{\mathrm{c}}, v^{\mathrm{uu}}, \mu\right) \in S^{1} \times B \times J\right\},
\end{aligned}
$$

where $B$ denotes a small ball in the infinite-dimensional space used to parametrize the strong stable and unstable fibers.

LEMMA 6.2. Under the above assumptions, there exist positive constants $L_{0}$ and $\eta$ such that the following is true for all $L>L_{0}$ and $\varphi \in S^{1}$ : there is a unique solution $v(x, \mu)$ of (6.5), defined for $x \in[-L, L]$, so that

$$
v(-L) \in \Sigma_{\text {in }}, \quad v(L) \in \Sigma_{\text {out }}, \quad v^{\mathrm{c}}(0)=\varphi,
$$

and $v(x)$ lies in a neighborhood of the periodic orbits $\gamma(x, \mu)$ for all $x \in[-L, L]$. Furthermore,

$$
\begin{aligned}
v(-L) & =\left(\varphi-L+\mathrm{O}\left(\mathrm{e}^{-\eta L}\right), \delta, v^{\mathrm{ss}}+\mathrm{O}\left(\mathrm{e}^{-\eta L}\right), \mathrm{O}\left(\mathrm{e}^{-\eta L}\right), \mathrm{O}\left(\mathrm{e}^{-\eta L}\right)\right), \\
v(L) & =\left(\varphi+L+\mathrm{O}\left(\mathrm{e}^{-\eta L}\right), \mathrm{O}\left(\mathrm{e}^{-\eta L}\right), \mathrm{O}\left(\mathrm{e}^{-\eta L}\right), \delta, v^{\mathrm{uu}}+\mathrm{O}\left(\mathrm{e}^{-\eta L}\right)\right), \\
v(0) & =\left(\varphi, \mathrm{O}\left(\mathrm{e}^{-\eta L}\right), \mathrm{O}\left(\mathrm{e}^{-\eta L}\right), \mathrm{O}\left(\mathrm{e}^{-\eta L}\right), \mathrm{O}\left(\mathrm{e}^{-\eta L}\right)\right) .
\end{aligned}
$$

The solution $v(x)$ is smooth in $(\varphi, \mu, L)$, and the error estimates in (6.7) can be differentiated. In addition,

$$
v(x,-\varphi)=\mathcal{R} v(-x, \varphi), \quad \varphi \in S^{1},|x| \leq L
$$

and, if Hypothesis 5 holds,

$$
v(x, \varphi+\pi)=\kappa v(x, \varphi), \quad \varphi \in S^{1},|x| \leq L .
$$

In particular, the solution $v(x, \varphi)$ is $\mathcal{R}$-reversible, with $v(0) \in \operatorname{Fix}(\mathcal{R})$, if and only if $\varphi=0, \pi$, and $\kappa \mathcal{R}$-reversible, with $v(0) \in \operatorname{Fix}(\kappa \mathcal{R})$, if and only if $\varphi=\pi / 2,3 \pi / 2$.

Once this lemma has been proven, analogies of Theorems 2.2 and 2.3 can be proven in a manner similar to the finite-dimensional setting, and we omit the details.

Proof. We cannot prove the statements within the zero-energy level set as flattening out this manifold destroys the semilinear nature of (6.5) which we need to apply the results on exponential dichotomies in [28]. Instead, we consider (6.5) in the full space $Y$.

Hypothesis 4 implies that there is a smooth two-parameter family $\gamma(\cdot, \mu, e)$ of symmetric periodic orbits that are parametrized by $\mu$ and their energy $e=\mathcal{H}(\gamma(0, \mu, e), \mu)$ for $e$ near zero. We normalize the family $\gamma(x, \mu, e)$ of periodic solutions so that $\gamma(0, \mu, e) \in \operatorname{Fix}(\mathcal{R})$ for all $(\mu, e)$. For fixed $(\mu, e, \varphi)$, we parametrize the strong stable fiber of $\gamma(x+\varphi, \mu, e)$ by $b^{+}$which incorporates the variable $v^{\mathrm{ss}}$ in the coordinates discussed before Lemma 6.2 and the time-like direction $x$. Thus, we denote by $q^{+}\left(x, \mu, e, \varphi, b^{+}\right)$the strong stable fiber of $\gamma(x+\varphi, \mu, e)$ so that $q^{+}$is smooth in all arguments and

$$
\left|\gamma(x+\varphi, \mu, e)-q^{+}\left(x, \mu, e, \varphi, b^{+}\right)\right| \leq K \mathrm{e}^{-\eta x}, \quad x \geq 0 .
$$

Similarly, $q^{-}\left(x, \mu, e, \varphi, b^{-}\right)$denotes the strong unstable fiber of $\gamma(x+\varphi, \mu, e)$. From now on, we denote various different constants by the letter $K$ : these different constants will be bounded by a uniform constant that does not depend on any of the parameters or variables involved. 

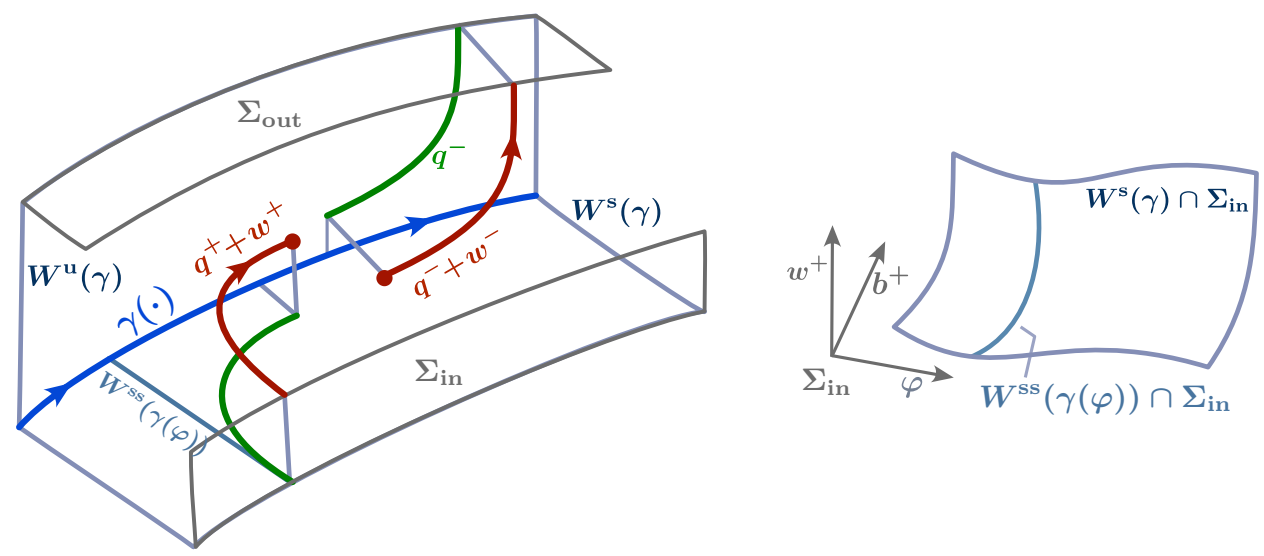

FIG. 6.1. A schematic picture of the matching procedure near the periodic orbit.

We seek solutions of the form

$$
\begin{aligned}
& u^{+}(x)=q^{+}\left(x, \mu, e^{+}, \varphi^{+}, b^{+}\right)+w^{+}(x), \quad x \in[0, L], \\
& u^{+}(0) \in \Sigma_{\text {in }}, \\
& u^{-}(x)=q^{-}\left(x, \mu, e^{-}, \varphi^{-}, b^{-}\right)+w^{-}(x), \quad x \in[-L, 0], \\
& u^{-}(0) \in \Sigma_{\text {out }}, \\
& u^{+}(L)=u^{-}(-L),
\end{aligned}
$$

and refer to Figure 6.1 for an illustration. Thus, $u^{ \pm}$satisfy (6.5) if and only if $w^{ \pm}$ satisfy

$$
w_{x}^{+}=\left[A(\mu)+N_{u}\left(q^{+}\left(x, \mu, e^{+}, \varphi^{+}, b^{+}\right)\right)\right] w^{+}+N^{+}\left(x, w^{+}\right), \quad 0 \leq x \leq L,
$$

where $N^{+}\left(x, w^{+}\right):=N\left(q^{+}+w^{+}\right)-N\left(q^{+}\right)-N_{u}\left(q^{+}\right) w^{+}=\mathrm{O}\left(\left|w^{+}\right|^{2}\right)$, and analogously for $w^{-}$. For the Floquet spectrum of the periodic orbits $\gamma(\cdot)$, we had assumed that there exists an $\eta>0$, independent of $(\mu, e)$, such that the real part of the spectrum, other than the two eigenvalues at zero, is bounded in absolute value from below by $\eta$. The results in [28] then imply that the linear part of (6.8) has exponential dichotomies $\Phi_{+}^{\mathrm{cs}}(x, y)$ and $\Phi_{+}^{\mathrm{u}}(x, y)$ that are smooth in $\left(\mu, e, \varphi^{+}, b^{+}\right)$such that, for $\epsilon>0$ sufficiently small, there exists a $K>0$ with

$$
\begin{aligned}
& \left|\Phi_{+}^{\mathrm{cs}}(x, y)\right|_{L(Y)} \leq K \mathrm{e}^{\epsilon(x-y)}, \quad x \geq y \geq 0, \\
& \left|\Phi_{+}^{\mathrm{u}}(x, y)\right|_{L(Y)} \leq K \mathrm{e}^{-\eta(y-x)}, \quad y \geq x \geq 0 .
\end{aligned}
$$

The variation-of-constants formula shows that solutions of $(6.8)$ on $[0, L]$ satisfy the integral equation

$$
\begin{aligned}
w^{+}(x)= & \Phi_{+}^{u}(x, L) a^{+}+\int_{L}^{x} \Phi_{+}^{\mathrm{u}}(x, y) N^{+}\left(y, w^{+}(y)\right) \mathrm{d} y \\
& +\int_{0}^{x} \Phi_{+}^{\mathrm{cs}}(x, y) N^{+}\left(y, w^{+}(y)\right) \mathrm{d} y \\
=: & {\left[\mathcal{F}\left(\mu, e^{+}, \varphi^{+}, a^{+}, b^{+}, w^{+}\right)\right](x) }
\end{aligned}
$$


and vice versa, where the quantity $a^{+} \in \operatorname{Rg}\left(\Phi_{+}^{\mathrm{u}}(L, L)\right)$ is an arbitrary element of the unstable fiber of $q^{+}$at $x=L$ and represents the final linear displacement off the center-stable manifold. For each fixed $\left(\mu, e^{+}, \varphi^{+}, a^{+}, b^{+}\right)$, we wish to view $\mathcal{F}$ as a smooth map from $C^{0}([0, L], Y)$, equipped with the weighted norm $\left\|w^{+}\right\|:=$ $\sup _{0 \leq x \leq L} \mathrm{e}^{\eta(L-x)}\left|w^{+}(x)\right|$, into itself. Indeed, $\mathcal{F}$ is well defined since

$$
\begin{aligned}
& \left\|\mathcal{F}\left(\mu, e^{+}, \varphi^{+}, a^{+}, b^{+}, w^{+}\right)\right\| \\
& \leq K\left|a^{+}\right|+\sup _{0 \leq x \leq L} K \mathrm{e}^{\eta(L-x)} \int_{L}^{x} \mathrm{e}^{-\eta(y-x)} \mathrm{e}^{-2 \eta(L-y)}\left\|w^{+}\right\|^{2} \mathrm{~d} y \\
& \quad+\sup _{0 \leq x \leq L} K \mathrm{e}^{\eta(L-x)} \int_{0}^{x} \mathrm{e}^{\epsilon(x-y)} \mathrm{e}^{-2 \eta(L-y)}\left\|w^{+}\right\|^{2} \mathrm{~d} y \\
& \leq K\left|a^{+}\right|+\sup _{0 \leq x \leq L} K\left[\mathrm{e}^{-\eta L} \int_{L}^{x} \mathrm{e}^{\eta y} \mathrm{~d} y+\mathrm{e}^{-\eta(L+x)} \mathrm{e}^{\epsilon x} \int_{0}^{x} \mathrm{e}^{(2 \eta-\epsilon) y} \mathrm{~d} y\right]\left\|w^{+}\right\|^{2} \\
& \leq K\left|a^{+}\right|+K\left\|w^{+}\right\|^{2},
\end{aligned}
$$

and it is also straightforward to verify that $\mathcal{F}$ is smooth in $\left(\mu, e^{+}, \varphi^{+}, a^{+}, b^{+}, w^{+}\right)$. In particular, for all sufficiently small $a^{+},(6.9)$ has a unique solution $w^{+}$, which satisfies $\left|w^{+}(x)\right| \leq K\left|a^{+}\right| \mathrm{e}^{-\eta(L-x)}$ for all $x \in[0, L]$. We also have

$$
\left|w^{+}(L)-a^{+}\right| \leq K\left|a^{+}\right|^{2}, \quad\left|w^{+}(0)\right| \leq K\left|a^{+}\right| \mathrm{e}^{-\eta L} .
$$

Analogous arguments apply to the function $w^{-}$, which satisfies the integral equation

$$
\begin{aligned}
w^{-}(x)= & \Phi_{-}^{\mathrm{s}}(x,-L) a^{-}+\int_{-L}^{x} \Phi_{-}^{\mathrm{s}}(x, y) N^{-}\left(y, w^{-}(y)\right) \mathrm{d} y \\
& +\int_{0}^{x} \Phi_{-}^{\mathrm{cu}}(x, y) N^{-}\left(y, w^{-}(y)\right) \mathrm{d} y
\end{aligned}
$$

for $x \in[-L, 0]$, where $a^{-} \in \operatorname{Rg}\left(\Phi_{-}^{\mathrm{s}}(-L,-L)\right)$ is in the stable fiber of $q^{-}$at $x=-L$. The unique solution of (6.10) satisfies

$$
\left|w^{-}(-L)-a^{-}\right| \leq K\left|a^{-}\right|^{2}, \quad\left|w^{-}(0)\right| \leq K\left|a^{-}\right| \mathrm{e}^{-\eta L} .
$$

We now solve the matching condition $u^{+}(L)=u^{-}(-L)$ given by

$$
\text { 1) } \gamma\left(L+\varphi^{+}, \mu, e^{+}\right)-\gamma\left(-L+\varphi^{-}, \mu, e^{-}\right)+a^{+}-a^{-}=\mathrm{O}\left(\left|a^{+}\right|^{2}+\left|a^{-}\right|^{2}+\mathrm{e}^{-\eta L}\right)
$$

where we used the fact that $\left|q^{+}\left(L, \mu, e^{+}, \varphi^{+}, b^{+}\right)-\gamma\left(L+\varphi^{+}, \mu, e^{+}\right)\right|=\mathrm{O}\left(\mathrm{e}^{-\eta L}\right)$ and analogously for $q^{-}$. To solve these equations, we fix $(\varphi, e)$ and set

$$
\begin{gathered}
e^{+}=e+\hat{e}, \quad \varphi^{+}=\varphi+\hat{\varphi}-L, \\
e^{-}=e-\hat{e}, \quad \varphi^{-}=\varphi-\hat{\varphi}+L
\end{gathered}
$$

for $(\hat{\varphi}, \hat{e})$ small, so that (6.11) becomes

$$
\gamma(\varphi+\hat{\varphi}, \mu, e+\hat{e})-\gamma(\varphi-\hat{\varphi}, \mu, e-\hat{e})+a^{+}-a^{-}=\mathrm{O}\left(\left|a^{+}\right|^{2}+\left|a^{-}\right|^{2}+\mathrm{e}^{-\eta L}\right) .
$$

Since the left-hand side vanishes at $\left(\hat{\varphi}, \hat{e}, a^{+}, a^{-}\right)=0$, and its derivative with respect to these variables is invertible, we find a locally unique solution $\left(\hat{\varphi}_{0}, \hat{e}_{0}, a_{0}^{+}, a_{0}^{-}\right)$of 
(6.12), which depends smoothly on the variables $\left(\mu, e, \varphi, b^{-}, b^{+}, L\right)$. We can also solve the remaining equations

$$
\begin{aligned}
& u^{+}(0)=q^{+}\left(0, \mu, e^{+}, \varphi^{+}, b^{+}\right)+\mathrm{O}\left(\mathrm{e}^{-\eta L} a^{+}\right) \in \Sigma_{\text {in }}, \\
& u^{-}(0)=q^{-}\left(0, \mu, e^{-}, \varphi^{-}, b^{-}\right)+\mathrm{O}\left(\mathrm{e}^{-\eta L} a^{-}\right) \in \Sigma_{\text {out }}
\end{aligned}
$$

for $\left(\hat{\varphi}_{0}, \hat{e}_{0}, a_{0}^{+}, a_{0}^{-}\right)$as functions of $\left(\mu, e, \varphi, b^{-}, b^{+}, L\right)$. We record that $\left(\hat{\varphi}_{0}, \hat{e}_{0}, a_{0}^{+}, a_{0}^{-}\right)=$ $\mathrm{O}\left(\mathrm{e}^{-\eta L}\right)$.

We determine now when these solutions are reversible. For given $\left(\mu, e, \varphi, b^{-}, b^{+}, L\right)$, we define

$$
v^{+}(x):=\mathcal{R} u^{-}(-x), \quad v^{-}(x):=\mathcal{R} u^{+}(-x),
$$

and note that these functions satisfy $(6.5)$ and

$$
\begin{aligned}
& v^{+}(L)=\mathcal{R} u^{-}(-L)=\mathcal{R} u^{+}(L)=v^{-}(-L) \\
& v^{+}(0)=\mathcal{R} u^{-}(0) \in \mathcal{R} \Sigma_{\text {out }}=\Sigma_{\text {in }} \\
& v^{-}(0)=\mathcal{R} u^{+}(0) \in \mathcal{R} \Sigma_{\text {in }}=\Sigma_{\text {out }}
\end{aligned}
$$

where we used that $u^{+}(L)=u^{-}(-L)$. We want to show that $v^{ \pm}=u^{ \pm}$whenever $\varphi \in\{0, \pi\}$ and $b^{-}=\mathcal{R} b^{+}$. For $\varphi=0$ and $b^{-}=\mathcal{R} b^{+}$, we have

$$
\begin{aligned}
& u^{+}(x)=q^{+}\left(x ; \mu, e+\hat{e}_{0}, \hat{\varphi}_{0}-L, b^{+}\right)+\Phi^{\mathrm{u}}(x, L) a_{0}^{+}+\hat{w}^{+}(x), \\
& u^{-}(x)=q^{-}\left(x ; \mu, e-\hat{e}_{0}, \hat{\varphi}_{0}+L, b^{-}\right)+\Phi^{\mathrm{s}}(x,-L) a_{0}^{-}+\hat{w}^{-}(x)
\end{aligned}
$$

for a unique $\left(\hat{\varphi}_{0}, \hat{e}_{0}, a_{0}^{+}, a_{0}^{-}\right)$, where all integrals present in (6.9) are subsumed into $\hat{w}^{ \pm}$. Hence,

$$
\begin{aligned}
& v^{+}(x)=\mathcal{R} u^{-}(-x)=q^{+}\left(x ; \mu, e-\hat{e}_{0},-\hat{\varphi}_{0}-L, b^{+}\right)+\Phi^{\mathrm{u}}(x, L) \mathcal{R} a_{0}^{-}+\mathcal{R} \hat{w}^{-}(x), \\
& v^{-}(x)=\mathcal{R} u^{+}(-x)=q^{-}\left(x ; \mu, e+\hat{e}_{0},-\hat{\varphi}_{0}+L, b^{-}\right)+\Phi^{\mathrm{s}}(x,-L) \mathcal{R} a_{0}^{+}+\mathcal{R} \hat{w}^{+}(x) .
\end{aligned}
$$

In the above, we have used $\mathcal{R} q^{+}\left(x ; \mu, e+\hat{e}_{0}, \hat{\varphi}_{0}-L, b^{+}\right)=q^{-}\left(x ; \mu, e+\hat{e}_{0},-\hat{\varphi}_{0}+\right.$ $L, \mathcal{R} b^{+}$) together with $b^{-}=\mathcal{R} b^{+}$, and similarly for $q^{-}$. We have shown above that $\left(\hat{\varphi}_{0}, \hat{e}_{0}, a_{0}^{+}, a_{0}^{-}\right)$, and therefore the functions $\hat{w}^{ \pm}$, are uniquely determined by $\left(\mu, \varphi, e, b^{+}, b^{-}, L\right)$. On the other hand, a comparison of the above expressions for $u^{ \pm}$ and $v^{ \pm}$shows that both $\left(\hat{\varphi}_{0}, \hat{e}_{0}, a_{0}^{+}, a_{0}^{-}\right)$and $\left(-\hat{\varphi}_{0},-\hat{e}_{0}, \mathcal{R} a_{0}^{-}, \mathcal{R} a_{0}^{+}\right)$give solutions for the same data $\left(\mu, \varphi, e, b^{+}, b^{-}, L\right)$ which means that $\hat{e}_{0}=\hat{\varphi}_{0}=0$ and $u^{ \pm}=v^{ \pm}$as claimed. This establishes that $u$ is reversible whenever $\varphi=0$ and $b^{-}=\mathcal{R} b^{+}$, and a similar argument gives reversibility when $\varphi=\pi$.

Finally, we need to show that we can choose $e$ so that the solutions $u^{ \pm}$lie in the zero energy set. Using that $\mathcal{H}$ is conserved along solutions, we find

$$
\begin{aligned}
\mathcal{H}\left(u^{+}(0)\right) & =\mathcal{H}\left(u^{+}(L)\right)=\mathcal{H}\left(q^{+}\left(L, \mu, e, \varphi-L, b^{+}\right)+\mathrm{O}\left(\mathrm{e}^{-\eta L}\right)\right) \\
& =\mathcal{H}\left(\gamma(\varphi, \mu, e)+\mathrm{O}\left(\mathrm{e}^{-\eta L}\right)\right)=\mathcal{H}(\gamma(0, \mu, e))+\mathrm{O}\left(\mathrm{e}^{-\eta L}\right)
\end{aligned}
$$

and therefore $\frac{\mathrm{d}}{\mathrm{de}} \mathcal{H}\left(u^{+}(0)\right) \neq 0$ near $e=0$ by hypothesis. Thus, we can solve near $e=0$ for the unique solution $e=e\left(\mu, \varphi, b^{+}, L\right)$ that will guarantee that $\mathcal{H}\left(u^{+}\right)=0$.

We are now in a position to transfer the results obtained in $Y$ to the flattened zero-energy level set by applying out the coordinate transformations described briefly before Lemma 6.2 , and we omit the details. 
6.4. Reversible systems without first integral. We assumed in Hypothesis 2 that the underlying ODE is conservative. For symmetric pulses, this assumption is not needed, and Theorem 2.2 is true for systems that satisfy only Hypotheses 1, 34, and 6-7. More precisely, we assume that there is a smooth two-parameter family $\gamma(\cdot, \mu, e)$ of symmetric periodic orbits that are parametrized by $\mu$ and a second internal parameter $e$. To find homoclinic orbits, we can utilize the Shilnikov variables that were discussed in the proof of Lemma 6.2 in $\S 6.3$ to characterize solutions that pass near the two-parameter family of symmetric periodic orbits. Symmetric orbits can then be found by following the proofs in $\S 4$ except that we now solve simultaneously not only for $(L, \mu)$ but also for the variable $e$ that encodes which of the reversible orbits $\gamma(\cdot, \mu, e)$ the homoclinic orbit follows.

To construct asymmetric pulses, we note that a first integral in $\mathbb{R}^{4}$, say, allows us to reduce each of the three-dimensional sections $\Sigma_{\text {in }}$ and $\Sigma_{\text {out }}$ to a two-dimensional section. If we do not have a first integral, we need two additional free variables to be able to solve in each section in the direction corresponding to $e$ along the oneparameter family $\gamma(x, \mu, e)$ of reversible periodic orbits. In one section, we can simply use the parameter $e$ itself. For non-conservative systems, no other free variables are available, and stationary asymmetric pulses should therefore not exist. Instead, asymmetric pulses should begin to move, and we are led to consider the Swift-Hohenberg equation in a moving frame $y=x-c t$,

$$
U_{t}=-\left(1+\partial_{y}^{2}\right)^{2} U+c \partial_{y} U-\mu U+\nu U^{2}-U^{3},
$$

and seek stationary localized states $U(y)$ for this equation, where $c$ provides now an additional free parameter. Using the unique periodic solution of the adjoint variational equation around each reversible periodic orbit, we find that the equation for $e$ in Fenichel's normal form becomes $e_{y}=c+$ h.o.t. Thus, we can use $e$ and $c$ to solve in the remaining directions in each section, and we conclude that asymmetric pulses exist exponentially close to the sets described by Lemma 5.3 for wave speeds that are $\mathrm{O}\left(\mathrm{e}^{-\eta L}\right)$.

7. Discussion. In this paper, we identified conditions on the global bifurcation structure of heteroclinic orbits between equilibria and periodic orbits that guarantee that we can glue connecting orbits together to construct both symmetric and asymmetric orbits that are homoclinic to the underlying equilibrium. We also showed that snaking occurs provided the global structure of fronts is as shown in Figure 1.5(i)(ii), while the structure sketched in Figure 1.5(iii) leads to infinitely many isolas of pulses. We reiterate that our results can be used to predict all branches of asymmetric pulses once the branches of the symmetric states have been computed: Figure 1.7 indicates how bifurcation branches can be constructed from the function $z$, while (1.5) shows that the branch $\mu_{*}\left(L, \varphi_{0}\right)$ of symmetric pulses is exponentially close to the graph $\mu=z\left(L+\varphi_{0}\right)$ of $z$, so that we can accurately calculate $z$ through numerical computations of the branch $\mu_{*}\left(L, \varphi_{0}\right)$ of symmetric pulses.

$P D E$ stability. We now comment on temporal stability of symmetric and asymmetric pulses in the context of self-adjoint partial differential equations (PDEs) such as the Swift-Hohenberg equation (1.1). Numerical computations of the first few eigenvalues of symmetric pulses of (1.1) have been published in [3, Figures 10-11], and our goal here is to compare these computations with theoretical predictions.

The spectrum of each front is the union of isolated eigenvalues and the essential spectra $\Sigma_{\text {ess }}^{0}$ and $\Sigma_{\text {ess }}^{\text {rolls }}$ of the trivial state and the asymptotic rolls, respectively. We assume that the front spectrum looks as shown in the top panel of Figure 7.1(i). In 


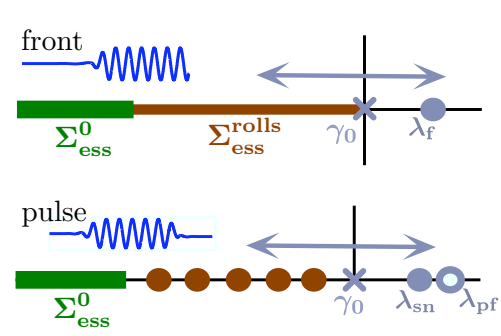

(i)

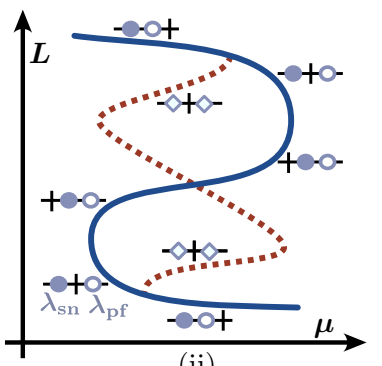

(ii)

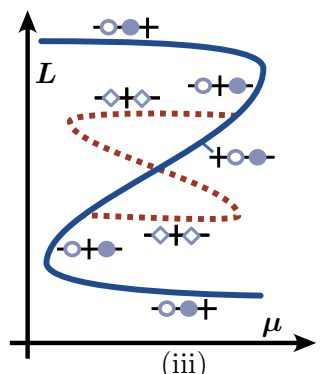

(iii)

FIG. 7.1. Panel (i) shows the anticipated PDE spectra of fronts [top] and symmetric 1-pulses [bottom]: $\Sigma_{\text {ess }}^{0}$ denotes the essential spectrum of the trivial state $U=0$; the essential spectrum $\Sigma_{\text {ess }}^{\text {rolls }}$ of the asymptotic rolls for fronts breaks up into $\mathrm{O}(L)$ eigenvalues for pulses; if the front has a single saddle-node eigenvalue, then we expect that pulses have two eigenvalues that move back and forth as we move along the snaking curve and cause saddle-node and pitchfork bifurcations. The expected movement of the saddle-node [solid circles] and pitchfork [open circles] eigenvalues is shown in more detail in panels (ii) and (iii), respectively corresponding to $g_{\mu}>0$ and $g_{\mu}<0$, along branches of symmetric [solid] and asymmetric [dotted] 1-pulses, where we assumed that symmetric pulses are stable along the lower branch.

particular, we assume that $\Sigma_{\text {ess }}^{0}$ lies on the negative real axis $\mathbb{R}^{-}$, at some distance away from zero, and that $\Sigma_{\text {ess }}^{\text {rolls }}$, which emerges from $\lambda=0$, lies in $\mathbb{R}_{0}^{-}$. The origin $\lambda=0$ is a branch point of the dispersion relation of the rolls. It remains to discuss the point spectrum of the fronts. Inspecting a typical bifurcation diagram such as Figure 1.5(i), we may expect that there is a single eigenvalue associated with the fronts that moves back and forth through zero at each fold bifurcation: note, however, that once the "eigenvalue" lies on the negative real axis, it is contained in $\Sigma_{\text {ess }}^{\text {rolls }}$, and there may no longer be a genuine eigenfunction associated with it. The Evans function ${ }^{5}$ provides a mechanism for identifying the saddle-node eigenvalue even when it lies in $\mathbb{R}_{0}^{-}$. The results in $[18,17]$ show that the Evans function $E(\gamma, \mu)$ is analytic in $\gamma$ near the origin ${ }^{6}$, where $\gamma^{2}=\lambda$, and that positive roots $\gamma$ of $E(\gamma, \mu)$ correspond to genuine positive eigenvalues $\lambda$, while negative roots $\gamma$ have only exponentially growing eigenfunctions. Thus, saddle-node eigenvalues can be tracked as zeros of $E$ even for $\lambda<0$. Typically, we expect that a root $\gamma$ crosses from $\mathbb{R}^{+}$to $\mathbb{R}^{-}$as we pass through a saddle-node bifurcation, and we therefore seem to lose the fold eigenvalue (as negative roots $\gamma$ do not correspond to genuine eigenvalues). However, if we take into account that $\gamma_{0}=0$ is a zero of the Evans function for all $\mu$ due to translation invariance, then a preliminary analysis yields the expansion $E(\gamma, \mu)=\gamma\left[a \gamma^{2}+b\left(\mu-\mu_{\mathrm{sn}}\right)+\right.$ h.o.t.], which implies that the fold root $\gamma$ moves from the positive axis onto the imaginary axis, and $\lambda=\gamma^{2}<0$ indeed becomes negative.

Next, we consider symmetric pulses along the snaking curve which are constructed by gluing together a front $U_{\mathrm{f}}(x)$ and its symmetric counterpart $U_{\mathrm{f}}(-x)$. We argue that the spectra of symmetric pulses should look as shown in the bottom panel of Figure 7.1(i). The essential spectrum of the linearization about a symmetric pulse is given by the spectrum $\Sigma_{\text {ess }}^{0}$ of $U=0$. The essential spectrum $\Sigma_{\text {ess }}^{\text {rolls }}$ of rolls is not in the spectrum of the pulses but instead breaks up into $\mathrm{O}(L)$ eigenvalues whose union converges to $\Sigma_{\text {ess }}^{\text {rolls }}$ in the symmetric Hausdorff distance as $L \rightarrow \infty[37,38,31]$.

\footnotetext{
${ }^{5}$ We refer to [36] for a survey

${ }^{6}$ To keep with standard notation, $\gamma$ stands for $\pm \sqrt{\lambda}$, and not for the periodic orbits, in this discussion
} 

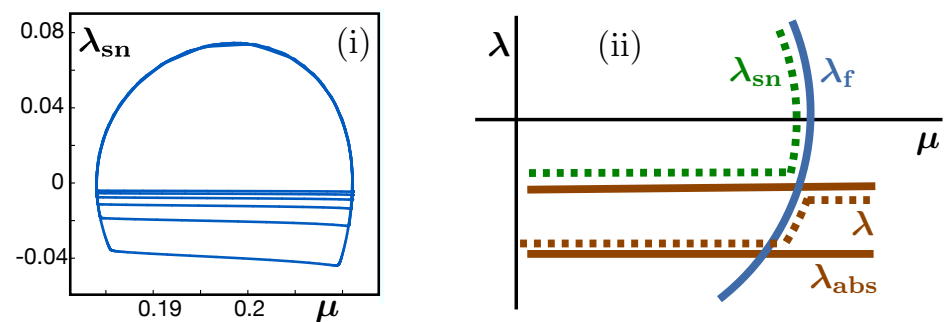

Fig. 7.2. Panel (i) shows the numerically computed saddle-node eigenvalue $\lambda_{\mathrm{sn}}$ of the symmetric pulses of (1.1) for $\nu=1.6$ along the snaking curve. Panel (ii) contains a schematic plot of the numerically computed saddle-node eigenvalue $\lambda_{\text {sn }}$ from panel (i) [upper-left dotted] and an absolute eigenvalue $\lambda$ [lower-right dotted], plotted together with the eigenvalue $\lambda_{\mathrm{f}}$ of the front [solid blue] and the theoretically predicted absolute eigenvalues $\lambda_{\text {abs }}$ [horizontal lines]. The computations in (i) suggest that the saddle-node eigenvalue becomes the first absolute eigenvalue once it reaches $\operatorname{Re} \lambda<0$.

Translation symmetry implies that the root $\gamma_{0}=0$ of the front persists as a simple eigenvalue of the pulses, which is in agreement with the numerical computations in [3, Figure 10].

For parameter values $\mu$ for which the front has an unstable saddle-node eigenvalue $\lambda_{\mathrm{f}}>0,[37]$ shows that symmetric pulses will have two eigenvalues, belonging to even and odd eigenfunctions, respectively, within $\mathrm{O}\left(\mathrm{e}^{-\eta L}\right)$ distance of $\lambda_{\mathrm{f}}$. At saddle-node and pitchfork bifurcations, these eigenvalues will cross through the origin onto the negative real axis, in agreement with the preceding discussion for fronts, where we asserted that the fold roots $\gamma \in \mathrm{i} \mathbb{R}$ of the Evans function correspond to elements $\lambda=\gamma^{2}<0$ on the negative axis. The fold and pitchfork eigenvalues will then collide with the $\mathrm{O}(L)$ absolute eigenvalues which emerge from the essential spectrum of the rolls and are situated at approximately $\mathrm{O}(1 / L)$-distance from each other. The spectral picture shown in [3, Figure 10] suggests that the saddle-node and pitchfork eigenvalues can be distinguished from these absolute eigenvalues. Our numerical computations, shown in Figure 7.2, indicate that this is not the case: instead, the saddle-node eigenvalue assumes the role of the first absolute eigenvalue. We do not know whether this is an artifact of the discretization or a genuine effect of the PDE operator but believe it is the latter.

Finally, we comment on the expected spectra of the asymmetric pulses that bifurcate at pitchfork bifurcations from the symmetric pulses. As illustrated in Figure 7.1(ii)-(iii), we expect asymmetric pulses to be unstable near onset, and we shall assume that symmetric pulses are stable along the lower branch. In the case of Figures 1.5(i) and 1.6(i), the asymmetric pulses do not undergo additional fold bifurcations and should therefore be unstable across the branch. In contrast, in the situation shown in Figures 1.5(ii) and 1.6(ii), the asymmetric pulses undergo additional folds as indicated in Figure 7.1(ii)-(iii), and the two critical eigenvalues are expected to be either both unstable or both stable along the middle branch.

Formal front interaction equations. To gain insight into the stability properties of asymmetric pulses, we discuss a formal approach in the spirit of collective coordinates, which attempts to capture the temporal interaction of the fronts that make up the pulses we constructed. We emphasize that this approach is, in the current context, 
entirely formal ${ }^{7}$. Starting point is the assumption that the underlying PDE

$$
U_{t}=-\left(1+\partial_{x}^{2}\right)^{2} U+F(U, \mu)
$$

has an invariant manifold consisting of possibly time-dependent pulse-like solutions of the type shown in the bottom panel of Figure 7.1(i). We parametrize this manifold by $U=U(L, \varphi)$, where $2 L$ is the spatial width of the roll plateau and $\varphi$ is the phase of the solution, relative to the rolls, at $x=0$. Substitution into the PDE gives

$$
U_{L} L_{t}+U_{\varphi} \varphi_{t}=-\left(1+\partial_{x}^{2}\right)^{2} U+F(U, \mu),
$$

where $(L, \varphi)$ depends only on $t$. In $\S 3-5$, we showed that stationary solutions of (7.1) with $\left(L_{t}, \varphi_{t}\right)=0$ are determined by the matching conditions

$$
\left(\begin{array}{c}
g(L+\varphi, \mu) \\
g(L-\varphi, \mu)
\end{array}\right)+\mathrm{O}\left(\mathrm{e}^{-\eta L}\right)=0
$$

which we derived in $\S 5$. If $\left(L_{t}, \varphi_{t}\right)$ is small, then the left-hand side of (7.1) is, for each fixed $t$, a small inhomogeneous perturbation of the ODE on the right-hand side, and we arrive at a matching condition similar to (7.2) which then uniquely determines $\left(L_{t}, \varphi_{t}\right)$; this is one way of deriving the pulse-interaction equations in $[10,45]$ and $[36$, $\S 5.3]$. In our situation, $\left(L_{t}, \varphi_{t}\right)$ is not small, and the resulting matching condition could be complicated. Without any rigorous justification, we assume that the contribution of the left-hand side of (7.1) to (7.2) is linear. Assuming furthermore, for simplicity, that $g(\varphi, \mu)=z(\varphi)-\mu$, then a comparison of the parametrization by $(L, \varphi)$ with (3.2) leads to the dynamical system

$$
\begin{aligned}
L_{t}+\varphi_{t} & =a[z(L+\varphi)-\mu]+\mathrm{O}\left(\mathrm{e}^{-\eta L}\right), \\
L_{t}-\varphi_{t} & =a[z(L-\varphi)-\mu]+\mathrm{O}\left(\mathrm{e}^{-\eta L}\right)
\end{aligned}
$$

for the temporal evolution of $(L, \varphi)$. Adding and subtracting the two equations in (7.3), we obtain

$$
\begin{aligned}
L_{t} & =\frac{a}{2}[z(L+\varphi)+z(L-\varphi)-2 \mu]+\mathrm{O}\left(\mathrm{e}^{-\eta L}\right), \\
\varphi_{t} & =\frac{a}{2}[z(L+\varphi)-z(L-\varphi)]+\mathrm{O}\left(\mathrm{e}^{-\eta L}\right) .
\end{aligned}
$$

In the Swift-Hohenberg equation, the trivial state invades rolls for all sufficiently large $\mu$, while rolls invade the background state for $\mu$ sufficiently small. This requires $a>0$, and we therefore focus on the equation

$$
\begin{aligned}
L_{t} & =z(L+\varphi)+z(L-\varphi)-2 \mu+\mathrm{O}\left(\mathrm{e}^{-\eta L}\right), \\
\varphi_{t} & =z(L+\varphi)-z(L-\varphi)+\mathrm{O}\left(\mathrm{e}^{-\eta L}\right),
\end{aligned}
$$

obtained by rescaling time in (7.4) for $a>0$. The linearization of (7.5) about an equilibrium has eigenvalues $\lambda_{ \pm}=z^{\prime}(L \pm \varphi)+\mathrm{O}\left(\mathrm{e}^{-\eta L}\right)$. In particular, we can read off the PDE eigenvalues predicted by this formal approach from the geometric construction of asymmetric pulses that we outlined in Figure 1.7. In the situation of Figure 7.1(ii)-(iii), these heuristic arguments predict that the asymmetric pulses are

\footnotetext{
${ }^{7}$ Situations in which interaction equations can be justified are discussed in [45] and the references therein
} 

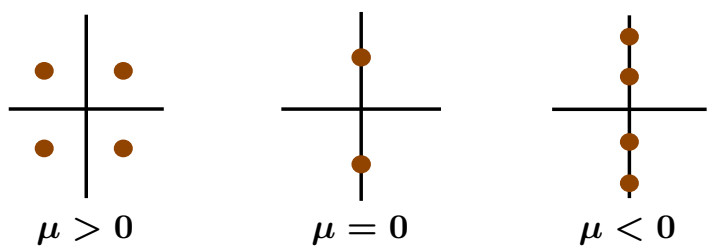

FIG. 7.3. Displayed are the spatial eigenvalues in the complex plane near a Hamiltonian Hopf bifurcation. This bifurcation corresponds to a Turing bifurcation, which occurs for $\mu=0$ in the Swift-Hohenberg equation.

stable on the middle branch, since we have $z^{\prime}(L \pm \varphi)<0$ along the middle branch, while they have one unstable eigenvalue on the other two branches in agreement with Figure 7.1(ii)-(iii).

The preceding formal analysis also predicts that symmetric pulses with $\varphi_{0} \in$ $\{0, \pi\}$ have two eigenvalues exponentially close to $z^{\prime}\left(L+\varphi_{0}\right)$, which agrees with the discussion above that led to Figure 7.1(i). The additional correlation between the derivative of the snaking curve and the fold and pitchfork eigenvalues of symmetric pulses is, at least qualitatively, reflected in the numerical computations of [3, Figure 10].

While the arguments outlined above are formal, we expect that our predictions for the location of eigenvalues of asymmetric pulses can be proved, or disproved, rigorously near each fold on the asymmetric bifurcation curves as we can utilize centermanifold reductions, but we have not yet pursued this further.

Multi-pulses. So far, we exclusively discussed pulses that follow the heteroclinic cycle formed by the front and its symmetric counterpart exactly once. In addition to these single-hump pulses, we expect to find multi-pulses that follow the heteroclinic cycle $N$-times for each $N \geq 2$, and the question is then whether these multi-pulses snake as well. The numerical computations in [41, 43], and more recently in [6, 20], suggest that multi-pulses actually lie on isolas. The techniques employed in this paper can also be used to study multi-pulses, and we currently carry this analysis out.

The Swift-Hohenberg equation, and degenerate Hamiltonian Hopf bifurcations. We now return to the steady-state equation

$$
\left(1+\partial_{x}^{2}\right)^{2} U+\mu U-\nu U^{2}+U^{3}=0
$$

of the Swift-Hohenberg equation. It is known that rolls bifurcate from $U=0$ in a Turing bifurcation, which occurs for $\mu=0$ and is supercritical for $\nu<\nu_{*}:=$ $\sqrt{27 / 38}$ and subcritical for $\nu>\nu_{*}$. For the first-order ODE associated with (7.6), this bifurcation corresponds to a Hamiltonian Hopf bifurcation, where two pairs of eigenvalues collide and split on the imaginary axis as shown in Figure 7.3. It was shown in [44] that the normal form of the degenerate Hamiltonian Hopf bifurcation near $\nu_{*}=\sqrt{27 / 38}$ has a heteroclinic cycle that connects $u=0$ to a periodic orbit along a branch $\nu=\nu_{\mathrm{M}}(\mu)$ in parameter space that emerges from $(\mu, \nu)=\left(0, \nu_{*}\right)$. Since the normal form is integrable and admits an $S^{1}$-symmetry, these heteroclinic orbits are degenerate in the sense that the two-dimensional unstable manifold of $u=0$ coincides with the two-dimensional stable manifold of the periodic orbit in the zero energy level set. In other words, the function $g(\varphi, \mu)$ from Figure 1.4 is identically zero, and so is the resulting function $z(\varphi)$ : snaking does not occur in the normal form as the solution branch is vertical. However, the normal form does not represent the full equation, which cannot be transformed into normal form. 
Chapman and Kozyreff $[21,7]$ recently investigated the existence of fronts and of symmetric and asymmetric pulses of (7.6) along the branch $\nu=\nu_{\mathrm{M}}(\mu)$ for $0<\mu \ll$ 1 near the degenerate Hamiltonian Hopf bifurcation through a formal asymptoticsbeyond-all-orders analysis. In their analysis, they fixed $0<\mu \ll 1$ and showed that, as $\nu$ is increased from a value below to a value above $\nu_{\mathrm{M}}(\mu)$, two fronts are first created and subsequently destroyed; the width of the associated existence interval is exponentially small in $\mu$. Chapman and Kozyreff then constructed symmetric and asymmetric pulses by gluing these fronts together. In particular, they showed that symmetric pulses of (7.6) with a roll plateau of length $2 L$ exist for $\nu$ given by

$$
\nu(\mu, L)=\nu_{\mathrm{M}}(\mu)+\frac{a_{0} \mathrm{e}^{-\frac{\pi}{\sqrt{\mu}}}}{\mu} \cos \left(L+\varphi_{0}+\frac{\log \mu}{4 \sqrt{734}}+a_{1}\right)+\mathrm{O}\left(\sqrt{\mu} \mathrm{e}^{-\sqrt{\mu} L}\right)
$$

for constants $a_{0}, a_{1} \in \mathbb{R}$ and with $\varphi_{0} \in\{0, \pi\}$ and $L \gg 1 / \mu$, see ${ }^{8}$ [7, Eqn. (162)]. They also showed that the two snaking branches are connected by ladder branches corresponding to asymmetric pulses. These results are established by a careful multiscale analysis: To any polynomial order in $(\mu, \nu)$, the phase of the Turing patterns that emerge from $\mu=0$ is not visible in the amplitude equations that describe slow modulations of the Turing patterns; this decoupling creates a one-parameter family of degenerate fronts in each truncated amplitude equation, which correspond to the degenerate heteroclinic orbits found in the normal form mentioned above. However, using the analyticity of (7.6), Chapman and Kozyreff showed how the exponentially small coupling of the fast phase to the slow modulation can be recovered through the calculation of Stokes curves of front and pulse solutions when the independent variable $x$ is moved into the complex plane. In summary, the beyond-all-orders analysis for $(7.6)$ in $[21,7]$ establishes that the function $z(\varphi)$, which describes fronts, is given by

$$
z(\varphi)=\nu_{\mathrm{M}}(\mu)+\frac{a_{0} \mathrm{e}^{-\frac{\pi}{\sqrt{\mu}}}}{\mu} \cos \left(\varphi+\frac{\log \mu}{4 \sqrt{734}}+a_{1}\right)
$$

and that symmetric pulses exist along two snaking branches, while asymmetric pulses exist on ladder branches that connect the snaking branches; the width of all branches is exponentially small in $\mu$.

In contrast, for our results, we need to assume that the function $z(\varphi)$ is not degenerate: this assumption then allows us to construct the bifurcation diagrams of symmetric and asymmetric pulses. Thus, if we assume that $z(\varphi)$ is given by (7.8), as shown by the formal analysis in [21,7], then Theorem 2.2 also gives (7.7). Indeed, we argued in $\S 4$ that Hypotheses 1-3 are satisfied for (7.6). Furthermore, Hypothesis 4 can be checked easily using the amplitude equations near the Hamiltonian Hopf bifurcation, see [44]. Applying Theorem 2.2 to (7.6) for fixed $\mu$ with $0<\mu \ll 1$ and varying $\nu$, we obtain from (2.7) and (7.8) the bifurcation equation

$$
\begin{aligned}
\nu & =z\left(L+\varphi_{0}\right)+\mathrm{O}\left(\mathrm{e}^{-\eta(\mu) L}\right) \\
& =\nu_{\mathrm{M}}(\mu)+\frac{a_{0} \mathrm{e}^{-\frac{\pi}{\sqrt{\mu}}}}{\mu} \cos \left(L+\varphi_{0}+\frac{\log \mu}{4 \sqrt{734}}+a_{1}\right)+\mathrm{O}\left(\mathrm{e}^{-\eta(\mu) L}\right)
\end{aligned}
$$

\footnotetext{
${ }^{8}$ The relation between the parameters used in [7] and those in (7.6) is explained in [7, Footnote 1 on p. 321]. The full width of the localized states constructed in [7] is given by $L / \epsilon^{4}$ in the notation of $[7]$ (see [7, Figure 3]), while we measure width relative to the phase $\varphi \in[0,2 \pi]$ of the rolls: this phase is given in [7, Eqn. (161)], and substitution of this equation into [7, Eqn. (162)] and using $L$ instead of $\varphi$ gives (7.7). Finally, the constant $\beta$ in [7, Eqn. (162)] is defined in [7, Eqn. (76)].
} 
for some $\eta(\mu)>0$, where $\varphi_{0}=0, \pi$. Since the Floquet multipliers $\pm \alpha(\mu)$ of the roll patterns are of order $\sqrt{\mu}$ for all $\nu$, our proof of Theorem 2.2 shows that we may take $\eta(\mu)=\mathrm{O}(\sqrt{\mu})$. The $x \mapsto-x$ symmetry of (7.6) implies that the two branches for $\varphi_{0}=0, \pi$ correspond to even localized states: solutions on the first branch have a maximum at $x=0$, while solutions on the second branch have a minimum at $x=0$. Similarly, the cubic-quintic Swift-Hohenberg equation (1.2) will exhibit two branches of symmetric pulses: one branch corresponds to even solutions $U(x)$ and their symmetric counterparts $-U(x)$, while the other branch consists of odd solutions and their symmetric counterparts.

To summarize the differences between our approach and that of [21, 7], their results establish the existence of fronts and of localized pulses for the Swift-Hohenberg equation (7.6) near the degenerate Turing (or Hamiltonian Hopf) bifurcation that occurs at $(\mu, \nu)=(0, \sqrt{27 / 38})$; it does not seem possible to extend them to degenerate Turing bifurcations in systems or to equations that are not analytic. Our results, on the other hand, establish the existence of localized pulses based on the assumption that the bifurcation structure of fronts is known: However, our results are not restricted to the vicinity of Hamiltonian Hopf bifurcations and infer the solution structure of symmetric and asymmetric localized pulses from the possibly very complex bifurcation structure of fronts; in addition, our results apply to non-conservative equations, to systems, and to problems posed on multidimensional cylindrical domains.

Finally, we mention that the theory presented here agrees with the earlier numerical results for both symmetric and asymmetric pulses that are summarized in Figure 1.1. Isolas of symmetric pulses were found in [3, Figure 24], which is in agreement with Theorem 6.1. Structures that are localized in one direction and periodic in the other direction were found recently in [1] for the planar Swift-Hohenberg equation. The numerically computed snaking curve resembles Figure 1.5(ii), and Theorem 2.3 predicts several interesting branches of asymmetric states, see Figure 1.6(ii). The conjecture stated above asserts that the bifurcating asymmetric solutions are stable along the middle branch. Subsequent to our analysis, we have computed the predicted asymmetric states and their PDE spectra [1], and these initial computations indicate that solutions are indeed stable on the middle branch.

\section{REFERENCES}

[1] D. Avitabile, J. Burke, E. Knobloch, D. J. B. Lloyd, And B. Sandstede, To snake or not to snake. In preparation, 2009.

[2] R. E. Beardmore, M. A. Peletier, C. J. Budd, and M. A. Wadee, Bifurcations of periodic solutions satisfying the zero-Hamiltonian constraint in reversible differential equations, SIAM J. Math. Anal., 36 (2005), pp. 1461-1488.

[3] J. Burke And E. KNOBLOCH, Localized states in the generalized Swift-Hohenberg equation, Phys. Rev. E, 73 (2006), 056211.

[4] - Homoclinic snaking: structure and stability, Chaos, 17 (2007), 037102.

[5] - Snakes and ladders: localized states in the Swift-Hohenberg equation, Phys. Lett. A, 360 (2007), pp. 681-688.

[6] — Multipulse states in the Swift-Hohenberg equation. Preprint, 2008.

[7] S. J. Chapman and G. KozyrefF, Exponential asymptotics of localized patterns and snaking bifurcation diagrams., Phys. D, 238 (2009), pp. 319-354.

[8] P. Coullet, C. Riera, and C. Tresser, Stable static localized structures in one dimension, Phys. Rev. Lett., 84 (2000), pp. 3069-3072.

[9] J. H. P. DAWES, Localized pattern formation with a large scale mode: slanted snaking, SIAM J. Appl. Dynam. Syst., 7 (2008), pp. 186-206.

[10] C. Elphick, E. Meron, and E. A. Spiegel, Patterns of propagating pulses, SiAM J. Appl. Math., 50 (1990), pp. 490-503. 
[11] N. Fenichel, Geometric singular perturbation theory for ordinary differential equations, J. Differ. Eqns., 31 (1979), pp. 53-98.

[12] W. J. Firth, L. Columbo, and A. J. Scroggie, Proposed resolution of theory-experiment discrepancy in homoclinic snaking, Phys. Rev. Lett., 99 (2007), 104503.

[13] D. Gomila, A. J. Scroggie, And W. J. Firth, Bifurcation structure of dissipative solitons, Phys. D, 227 (2007), pp. 70-77.

[14] M. F. Hilali, S. Metens, P. Borckmans, and G. Dewel, Pattern selection in the generalised Swift-Hohenberg model, Phys. Rev. E, 51 (1995), pp. 2046-2052.

[15] G. W. Hunt, G. J. Lord, And A. R. Champneys, Homoclinic and heteroclinic orbits underlying the post-buckling of axially-compressed cylindrical shells, in Localization and solitary waves in solid mechanics, World Sci. Publ., River Edge, 1999, pp. 285-297.

[16] G. W. Hunt, M. A. Peletier, A. R. Champneys, P. D. Woods, M. A. Wadee, C. J. Budd, AND G. J. LorD, Cellular buckling in long structures, Nonlin. Dynam., 21 (2000), pp. 3-29.

[17] T. Kapitula AND J. Rubin, Existence and stability of standing hole solutions to complex Ginzburg-Landau equations, Nonlinearity, 13 (2000), pp. 77-112.

[18] T. Kapitula And B. Sandstede, Stability of bright solitary-wave solutions to perturbed nonlinear Schrödinger equations, Phys. D, 124 (1998), pp. 58-103.

[19] J. KNobloch And T. WAGENKNecht, Homoclinic snaking near a heteroclinic cycle in reversible systems, Phys. D, 206 (2005), pp. 82-93.

[20] - Snaking of multiple homoclinic orbits in reversible systems, SIAM J. Appl. Dynam. Syst., 7 (2008), pp. 1397-1420.

[21] G. Kozyreff and S. J. Chapman, Asymptotics of large bound states of localized structures, Phys. Rev. Lett., 97 (2006), 044502.

[22] M. Krupa, B. Sandstede, and P. Szmolyan, Fast and slow waves in the FitzHugh-Nagumo equation, J. Differ. Eqns., 133 (1997), pp. 49-97.

[23] D. J. B. Lloyd, B. Sandstede, D. Avitabile, and A. R. Champneys, Localized hexagon patterns of the planar Swift-Hohenberg equation, SIAM J. Appl. Dynam. Syst., 7 (2008), pp. 1049-1100.

[24] G. J. Lord, A. R. Champneys, And G. W. Hunt, Computation of homoclinic orbits in partial differential equations: an application to cylindrical shell buckling, SIAM J. Sci. Comput., 21 (1999), pp. 591-619.

[25] A. Mielke, A spatial center manifold approach to steady-state bifurcations from spatially periodic patterns, in Dynamics of nonlinear waves in dissipative systems, G. Dangelmayr, B. Fiedler, K. Kirchgässner, and A. Mielke, eds., Pitman Research Notes in Mathematics, Longman, Harlow, 1996, pp. 209-262.

[26] - Instability and stability of rolls in the Swift-Hohenberg equation, Comm. Math. Phys., 189 (1997), pp. 829-853.

[27] Y. Nishiura And D. Ueyama, A skeleton structure of self-replicating dynamics, Phys. D, 130 (1999), pp. 73-104.

[28] D. Peterhof, B. Sandstede, and A. Scheel, Exponential dichotomies for solitary-wave solutions of semilinear elliptic equations on infinite cylinders, J. Differ. Eqns., 140 (1997), pp. 266-308.

[29] Y. Pomeau, Front motion, metastability, and subcritical bifurcations in hydrodynamics, Phys. D, 23 (1986), pp. 3-11.

[30] J. D. M. RADEMACHER, Homoclinic orbits near heteroclinic cycles with one equilibrium and one periodic orbit, J. Differ. Eqns., 218 (2005), pp. 390-443.

[31] _ Geometric relations of absolute and essential spectra of wave trains, SIAM J. Appl. Dynam. Syst., 5 (2006), pp. 634-649.

[32] H. Sakaguchi And H. R. BRAnd, Stable localized solutions of arbitrary length for the quintic Swift-Hohenberg equation, Phys. D, 97 (1996), pp. 274-285.

[33] _ Stable localized squares in pattern-forming nonequilibrium systems, Europhys. Lett., 38 (1997), pp. 341-346.

[34] - Localized patterns for the quintic complex Swift-Hohenberg equation, Phys. D, 117 (1998), pp. 95-105.

[35] B. SAndstede, Verzweigungstheorie homokliner Verdopplungen, $\mathrm{PhD}$ thesis, University of Stuttgart, 1993.

[36] - Stability of travelling waves, in Handbook of dynamical systems, Vol. 2, North-Holland, Amsterdam, 2002, pp. 983-1055.

[37] B. Sandstede And A. Scheel, Absolute and convective instabilities of waves on unbounded and large bounded domains, Phys. D, 145 (2000), pp. 233-277.

[38] — Gluing unstable fronts and backs together can produce stable pulses, Nonlinearity, 13 (2000), pp. 1465-1482. 
[39] — Defects in oscillatory media: toward a classification, SIAM J. Appl. Dynam. Syst., 3 (2004), pp. 1-68.

[40] S. Schecter, Exchange lemmas I: Deng's lemma, J. Differ. Eqns., 245 (2008), pp. 392-410.

[41] G. H. M. van der Heijden, A. R. Champneys, and J. M. T. Thompson, Spatially complex localisation in twisted elastic rods constrained to a cylinder, Internat. J. Solids Structures, 39 (2002), pp. 1863-1883.

[42] A. Vanderbauwhede and B. Fiedler, Homoclinic period blow-up in reversible and conservative systems, Z. Angew. Math. Phys., 43 (1992), pp. 292-318.

[43] M. K. Wadee, C. D. Coman, And A. P. Bassom, Solitary wave interaction phenomena in the strut buckling model incorporating restabilisation, Phys. D, 163 (2002), pp. 26-48.

[44] P. D. Woods And A. R. Champneys, Heteroclinic tangles and homoclinic snaking in the unfolding of a degenerate reversible Hamiltonian-Hopf bifurcation, Phys. D, 129 (1999), pp. $147-170$.

[45] S. ZeliK AND A. Mielke, Weak interaction in multi-pulse structures and space-time chaos in reaction-diffusion equations, Mem. Amer. Math. Soc. (to appear). 